\title{
Rapid distortion analysis and direct simulation of compressible homogeneous turbulence at finite Mach number
}

\author{
By C. Cambon, ${ }^{1}$ G. N. Coleman ${ }^{2}$ AND N. N. Mansour ${ }^{3}$
}

The effect of rapid mean compression on compressible turbulence at a range of turbulent Mach numbers is investigated. Rapid distortion theory (RDT) and direct numerical simulation results for the case of axial (one-dimensional) compression are used to illustrate the existence of two distinct rapid compression regimes. These regimes are set by the relationships between the timescales of the mean distortion, the turbulence, and the speed of sound. A general RDT formulation is developed and is proposed as a means of improving turbulence models for compressible flows.

\section{Introduction}

This paper focuses upon the behavior of homogeneous compressible turbulence under the influence of rapid axial (one-dimensional) mean compression. The motivation for this study is a need to cast light upon the physics of compressible turbulent flows and to improve compressible turbulence models. Our approach is to use both direct numerical simulations (DNS) and rapid distortion theory (RDT). The RDT developed in this paper is for general (those that preserve homogeneity) mean deformations; the resulting insight is then used to suggest improvements to compressible turbulence models that are applied to rapidly compressed flows.

Earlier RDT studies of homogeneous compressible turbulence have been limited to either isotropic compressions (Blaisdell 1992, private communication) or the vanishing turbulent Mach number limit (Durbin \& Zeman 1992, hereafter referred to as DZ); the present investigation, therefore, attempts a more general treatment in that non-isotropic compressions and finite Mach numbers are considered. Some of our main conclusions confirm and extend those found in the recent study of shockturbulence interactions by Jacquin \& Cambon (1992).

An overview of our findings follows. The RDT analysis predicts that the crucial parameter for turbulence subjected to rapid compression is the ratio of the mean deformation rate, $D$, to the inverse sonic timescale $L / a$, where $L$ is a turbulent lengthscale and $a$ is the sound speed. This parameter, $D L / a$, hereinafter denoted as $\Delta m$ (after $\mathrm{DZ}$ ), is equivalent to the product of the inverse of the turbulent timescale, the deformation rate, and the turbulent Mach number, $M_{i} ;$ it defines for the dilatational part of the velocity field two distinct limits: the "pseudo-acoustical" 
(nearly solenoidal) regime given by $\Delta m \ll 1$ (that studied by DZ) and the so-called "pressure-released" regime with $\Delta m \gg 1$. The term "pressure-released" is chosen because when $\Delta m$ is large, the sonic and turbulent timescales are both much larger than $D^{-1}$ and, therefore, correlations involving the fluctuating pressure and velocity fields are negligible during a rapid distortion. The behavior of the solenoidal velocity field, according to the RDT analysis, is unaffected by the dilatational field when the mean flow is irrotational, and is thus independent of $\Delta m$ for axial compressions. Its history is, therefore, identical to that predicted for compression of purely solenoidal turbulence. In the following, we confirm these RDT predictions by comparison with DNS results.

The DNS results also show that for moderate values of $\Delta m$, all the double-velocity correlations involving the dilatational part of the turbulent velocity field remain weak with respect to the pure-solenoidal correlations and are in this sense similar to the pure solenoidal case, even for moderate compressibility. Only the $\Delta m \gg 1$ case is characterized by a strong amplification of the dilatational correlations.

The moderate $\Delta m$ results are at first glance in conflict with recent studies of axially compressed turbulence (e.g., DZ, Zeman \& Coleman 1992) which find unexpectedly large pressure-dilatation correlations in the nearly solenoidal flow. This led us to investigate the behavior of the pressure field, which has two roles for a rapid compression. On one hand, it modifies the production term in the turbulent kinetic energy equation by changing the Reynolds stress anisotropy through the classic pressure-strain rate correlation (via $\Pi_{11}$ for an axial compression in the $x_{1}$ direction). On the other hand, the pressure is directly involved in the kinetic energy equation through the pressure-dilatation term, $\Pi=\Pi_{i i} / 2$. The magnitude of $\Pi_{11}$ is found to be larger than that of $\Pi$ in all cases considered in this paper for a wide range of Mach numbers and large (but finite) compression speeds.

Both the pressure variance and pressure-dilatation correlation from the DNS are found to increase with Mach number (and, therefore, with $\Delta m$ at a fixed mean distortion-to-turbulent timescale ratio) with respect to their initial values. However, when $\Pi$ is compared to the production term in the turbulent kinetic energy transport equation, it is much smaller and has, in fact, less relative importance with increasing $M_{t}$. This reduced relative importance of the pressure field with increasing compressibility is a key result of this paper and is the basis of much of what follows. Between the $\Delta m \rightarrow 0$ and $\Delta m \rightarrow \infty$ extremes (where the pressuredilatation correlation is identically zero), $\Pi$ must reach a maximum; from the DNS results, it appears that this maximum occurs near the $\Delta m \rightarrow 0$ limit at a small but finite value.

In the next section, the RDT analysis is developed for compressible homogeneous turbulence; in $\S 3$, the theory is applied to the case of axial compression, and separate analytic expressions for the relevant dilatational and solenoidal correlations for both the $\Delta m \ll 1$ and $\Delta m \gg 1$ extremes are presented and compared to DNS results. The findings suggest that it would be appropriate for turbulence models to "interpolate" between the two extremes in order to accurately capture the $M_{t}$ dependence during a rapid axial compression. We propose two methods for doing so 
in $\S 4$, which deals with the role of, and closures for, the pressure-strain rate correlation. Section 5 considers the implications of this study for isotropically compressed and sheared flows, and $\S 6$ contains a recap of the main results and our conclusions.

2. A rapid distortion analysis for compressible homogeneous turbulence

\subsection{General considerations}

Blaisdell et al. (1991, hereafter referred to as BMR) observed that the "intrinsic compressibility" (the non-zero divergence) of the turbulent field often tends to reduce the amplification of turbulent kinetic energy produced by a mean velocity gradient, such as a bulk compression or mean shear, with respect to the pure solenoidal case. This effect depends on at least three different timescales and on the initial turbulent field. These are the mean distortion timescale,

$$
\tau_{D}^{-1}=\left(U_{i, j} U_{j, i}\right)^{1 / 2}
$$

(where $U_{i, j}$ is the mean velocity gradient), the "turbulent decay" or "turn-over" time,

$$
\tau_{t}^{-1}=q / L
$$

(where $q^{2} / 2$ is the turbulent kinetic energy and $L$ is a lengthscale of the energy containing eddies), and the timescale linked to the sonic speed,

$$
\tau_{a}^{-1}=a / L \text {. }
$$

The compression speed, $r=\tau_{i} / \tau_{D}$, is the only relevant parameter for modeling homogeneous incompressible turbulence (at least for large Reynolds number). However, when intrinsic compressibility is considered, the ratio of the two latter timescales, which amounts to a turbulent Mach number $M_{t}=\tau_{a} / \tau_{t}$, must also be accounted for. The magnitude of the reduction of the kinetic energy amplification mentioned above is, therefore, not necessarily universal, given the multi-timescale and initial-value nature of the problem. In fact, RDT studies of inhomogeneous flows even go so far as to predict an increase with $M_{t}$ of the kinetic energy amplifcation for turbulence under rapid (but finite) compression; these studies by Debiève et al. (1982, hereafter referred to as DGG) and Jacquin \& Cambon (1992) are discussed in a following subsection, where the general RDT equations are presented and the reasons for the apparent growth rate versus $M_{t}$ discrepancy are given. This analysis is based on an extended Craya-Herring decomposition (Cambon 1982, 1990; Cambon et al. 1985), which is shown to facilitate a separate investigation of the solenoidal and dilatational histories and provides a useful comparison to other approaches (e.g., BMR and DZ).

Some of the earlier RDT studies have apparently over-estimated the role of the pressure-dilatation term, attempting to force an increased damping due to compressibility of the kinetic energy growth rate. We hope to clarify the situation here by separately considering various terms in one-point closure equations and thus use RDT as a tool for improving a model's representation of those terms. While the RDT is not a model in and of itself, by improving the accuracy of crucial terms, we expect that it will in turn also improve the overall accuracy of the model. 


\subsection{Definitions and background}

To investigate the influence of the mean flow upon the turbulence, it is convenient to use a coordinate system $x_{i}$ that deforms with the mean deformation. We accordingly define the Lagrangian displacement tensor $F_{i j}$ (Eringen 1967) via

$$
d x_{i}=\frac{\partial x_{i}}{\partial t} d t+\frac{\partial x_{i}}{\partial X_{j}} d X_{j}=U_{i} d t+F_{i j} d X_{j}
$$

where $x_{i}(\mathrm{X}, t)$ is the position at time $t$ of a fluid particle moving with the mean flow, which has the position $X_{i}$ at the initial time $t=0$. Representing the substantial time derivative by a superimposed dot, one has

$$
\dot{F}_{i j}=\frac{\partial \dot{x}_{i}}{\partial X_{j}}=\frac{\partial \dot{x}_{i}}{\partial x_{l}} \frac{\partial x_{l}}{\partial X_{j}}=U_{i, l} F_{l j} \quad \text { with } \quad F_{i j}(\mathbf{X}, t=0,0)=\delta_{i j}
$$

where

$$
()=(), t+U_{j} \frac{\partial()}{\partial x_{j}}
$$

is the substantial derivative; we shall also have occasion to use the symbol $\mathcal{D}() / \mathcal{D} t$ to denote the substantial derivative. Unless stated otherwise, the dependent variables are assumed to be decomposed into Reynolds averaged and fluctuating components, as $U_{i}+u_{i}$, where capital letters, overbars, and angle-brackets are all used interchangeably to denote Reynolds- (ensemble) averaged quantities, and either lowercase or primed variables are used to denote fluctuating quantities. Note that $\mathbf{F}$ is a function of the stationary coordinate $\mathbf{X}$, the time $t$, and is parameterized by the time (in units of $t$ ) at which the tensor is orthonormal (hence the third argument in (5a)). For flows under mean compression, the determinant of $\mathbf{F}$ has special significance since it is equal to the volumetric ratio $J$.

When the mean velocity field is irrotational, the analyses proposed (over a hundred years ago!) by Cauchy, Weber, or Kelvin for the total (mean plus fluctuating) vorticity can be used to give solutions for the fluctuating vorticity $\left(\omega_{i}=\epsilon_{i j k} u_{k, j}\right)$ and velocity fields:

$$
\begin{gathered}
\omega_{i}(\mathbf{x}, t)=\frac{1}{J} F_{i j}(\mathbf{X}, t, 0) \omega_{j}(\mathbf{X}, 0) \\
u_{i}(\mathbf{x}, t)=F_{j i}^{-1}(\mathbf{X}, t, 0) u_{j}(\mathbf{X}, 0)+\phi_{, i} .
\end{gathered}
$$

These solutions, which ultimately derive from the linearized Euler equations, remain approximately valid for moderately inhomogeneous flows (recall the spatial dependence of F). Eq. (6) is the classic solution of the linearized Helmholtz equation when the mean vorticity-fluctuating velocity term is zero (that is, for an irrotational mean flow). When this term is not zero, simple solutions in physical space are not possible. Eq. (7) (also valid only for irrotational mean flows), an expression which has been extensively used by Goldstein (1978), contains the scalar potential $\phi$, which is directly connected to the fluctuating pressure and can be calculated once certain assumptions are made (e.g., that the fluctuating velocity field is solenoidal or that 
the dilatational field is nearly acoustic). The term $\phi$ is not the scalar potential arising from the Helmholtz decomposition (which we will denote $\varphi$ in the following) because the " $F_{j i}^{-1}$ " term in (7) contains contributions from both the solenoidal and dilatational velocity field.

DGG's RDT solution of the Lagrangian transport equation for the Reynolds stress tensor for the case of shock wave-turbulence interaction reads

$$
\left\langle u_{i} u_{j}\right\rangle\left(t^{+}\right)=F_{m i}^{-1}\left(t^{+}, t^{-}\right)\left(u_{m} u_{n}\right\rangle\left(t^{-}\right) F_{n j}^{-1}\left(t^{+}, t^{-}\right),
$$

where $t^{-}$and $t^{+}$refer to positions upstream and downstream of the shock, respectively, following a mean streamline through the shock. The shock is considered as a pure discontinuity of the mean streamwise velocity. In other words, it is an external streamwise compression of infinite rate, and the associated tensor $F$ does not depend on the history of the velocity gradient, but is completely characterized by the mean density jump or mean volumetric ratio $J=\operatorname{Det}(\mathbf{F})$, with $F_{i j}=J \delta_{i 1} \delta_{j 1}$ through the shock. The ratio $J$ is linked to the upstream Mach number $M_{0}$ via

$$
J=\frac{2+(\gamma-1) M_{0}^{2}}{(\gamma+1) M_{0}^{2}}
$$

where $\gamma$ is the ratio of specific heats, and use has been made of the classic RankineHugoniot relations for the mean (frozen) field. A comparison of equations (7) and (8) shows that this approach ignores the effect of pressure (which is mediated by $\phi$ in (7)); the response of the pressure fluctuations with a finite characteristic time even for the so-called "rapid" term is neglected compared to an infinite compression rate). Another idealization in the analysis of DGG, also pointed out by Lee et al. (1992), is that the distortion (curvature and unsteadiness) of the shock surface by the impinging turbulent structure is ignored. The latter issue, initially addressed by Ribner(1953), is not considered by the present paper. We investigate instead the role of the pressure field in a simpler homogeneous framework by explicitly defining and formalizing the range of validity of the "pressure released" regime that is implicit in the Debiève analysis. This paper has much in common with the recent analysis of the shock wave flow performed by Jacquin \& Cambon (1992), in which the pressure-released limit was first explicitly advocated.

Equation (7) shows that an irrotational deformation of a purely solenoidal velocity field is given by

$$
u_{i}(\mathbf{x}, t)=u_{i}^{s}(\mathbf{x}, t)=\left(F_{j i}^{-1}(\mathbf{X}, t, 0) u_{j}(\mathbf{X}, 0)\right)^{s},
$$

where to maintain $u_{i, i}=0$ we have,

$$
\phi_{, i}=-\left(F_{j i}^{-1}(\mathbf{X}, t, 0) u_{j}(\mathbf{X}, 0)\right)^{d}
$$

(where the $s$ and $d$ superscripts (and later subscripts) are understood to respectively refer to the solenoidal and dilatational contributions). The latter equation is an integral form of the Poisson equation for the fluctuating pressure,

$$
\nabla^{2} \phi=-\left(F_{j i}^{-1} u_{j}\right)_{, i} .
$$


For the solenoidal case, the pressure "kills off" the dilatational contribution, resulting in the lower limit of the kinetic energy growth rate caused by the mean compression. Conversely, in the pressure-released regime, the $u_{i}^{d}$ contribution in Eq. (11) is no longer "removed" by the pressure, producing an extra contribution to the solenoidal energy, which is unaffected by the dilatation field and again grows in accordance with Eq. (10); in other words, the compressibility leads to an increase in the kinetic energy growth rate.

From this point hence, the RDT analysis will be continued under the assumption of flow homogeneity and make use of a spectral formalism; the Fourier wave-space proves to be invaluable for obtaining tractable RDT solutions. Beginning with Eq. (6), we shall use the Fourier space to extract the solenoidal velocity from the vorticity, as was done by Batchelor \& Proudman (1954). Instead of solving a Poisson equation in physical space, we use a simple geometric wave-space projection to invoke the Helmholtz decomposition.

\subsection{The mean flow}

Before turning to the turbulent fields, however, we restrict the types of mean deformations that are admitted by this analysis to those that preserve the homogeneity of the flow. In incompressible turbulence, the constraint of maintaining homogeneous statistical properties leads to two conditions: the mean velocity gradient $U_{i, j}$ must be uniform in space, and the mean flow must be a particular solution of the Navier-Stokes equations. The last condition amounts to an irrotational mean acceleration,

$$
\nabla \times \mathbf{\Gamma}=\mathbf{0}
$$

or that

$$
\dot{U}_{i, j}+U_{i, l} U_{i, j}
$$

is symmetric, where

$$
\Gamma_{i}=\left(\dot{U}_{i, j}+U_{i, l} U_{l, j}\right) x_{j}=\ddot{F}_{i j}(t, 0) X_{j}
$$

Compressibility introduces a new condition. The linearization of the momentum equation displays two acceleration terms. The first one is the product of mean density and the fluctuating acceleration and leads to the same constraint mentioned above. The second term is the product of density fluctuation $\rho$ ' by the mean acceleration $\Gamma$ and is typically nonhomogeneous (as can be seen by the spatial dependence in (14)). This term can be removed, and homogeneity preserved, by neglecting the density fluctuation with respect to the mean density. Such an approximation (which is consistent with "compressed" turbulence at low Mach number) will be not used in this paper. Instead, we admit only mean flows without convective acceleration. From eqs. (5) and (14) we see that this requires

$$
F_{i j}(t, 0)=\delta_{i j}+A_{i j} t
$$

or

$$
U_{i, j}(t)=A_{i l} F_{l j}^{-1}=A_{i l}\left(\delta_{l j}+A_{l j} t\right)^{-1}
$$


Eq. (15) is valid for an arbitrary constant (not necessarily symmetric) matrix $\mathbf{A}$ for arbitrary times, provided that the determinant of F, $J$, remains positive. Special cases of (15) have been given previously, for example, for pure strain and shear (BMR). A good approximation for the mean pressure $P$ as a function of $J$ can be derived from the isentropic relations. (While the isentropic relations are not strictly valid when $M_{t}$ is nonzero, DNS of finite $M_{t}$ turbulence under mean compression have shown that the deviation from the isentropic prediction is relatively small: for example, in Case C1DV discussed below - a rapid axial compression for initial $M_{t}=0.3$ - the mean pressure at $J=\bar{\rho}(0) / \bar{\rho}=1 / 5$ is within 6 percent of the isentropic value.)

\subsection{The fluctuating flow}

The linearized Euler equations (with $\rho^{\prime} \Gamma_{i}=0$ ) in the deforming coordinate system are

$$
\dot{u}_{i}+U_{i, j} u_{j}=-\frac{p_{, i}}{\bar{\rho}}
$$

with $\bar{\rho}=\bar{\rho}(t)=\bar{\rho}(0) / J(t)$ (recall that the dot superscript denotes a substantial derivative, see Eq. (5)). The linearized equations for the fluctuating pressure $p$ and entropy $s$ read (see DZ)

$$
\begin{gathered}
\left(\frac{\dot{p}}{\gamma P}\right)=-u_{i, i} \\
\dot{s}=0,
\end{gathered}
$$

where $P=\bar{\rho} R T$. An investigation of the coupling between solenoidal and dilatational contributions to the fluctuating velocity field is conveniently done by transforming the variables in (the deformed coordinate) $\mathbf{x}$ into (three-dimensional) Fourier space, which we indicate either by a caret symbol or the notation " $\hat{\mathcal{F}}()$." The classic Helmholtz decomposition is given first in physical and then in spectral space as follows:

$$
\begin{gathered}
v_{i}(\mathbf{x}, t)=\epsilon_{i j l} \psi_{l, j}+\varphi_{, i} \\
\widehat{v}_{i}(\mathbf{k}, t)=\left(\delta_{i j}-\frac{k_{i} k_{j}}{k^{2}}\right) \widehat{v}_{j}+\frac{k_{i} k_{j}}{k^{2}} \widehat{v}_{j},
\end{gathered}
$$

for any vector field $\mathbf{v}$. The two terms on the right-hand sides correspond to $\mathbf{v}^{\mathbf{s}}$ and $\mathbf{v}^{d}$, which are defined in physical space by the vector $\psi_{i}$ and the scalar potential $\varphi$. The corresponding spectral space decomposition into $\widehat{\mathbf{v}}^{s}$ and $\widehat{\mathbf{v}}^{d}$ is given by the projection operators in (19), which separate the (single-component) dilatational contribution parallel to the wavevector $\mathbf{k}$ from the (two-component) solenoidal contribution in the plane normal to k. Equations (16) and (17) are easily Fouriertransformed; only the advection term requires particular caution:

$$
\widehat{\dot{u}}_{i}=\hat{\mathcal{F}}\left(u_{i, l}+U_{j, l} x_{l} u_{i, j}\right),
$$

so

$$
\hat{\dot{u}}_{i}=\widehat{u}_{i, t}-U_{l, l} \widehat{u}_{i}-U_{j, l} k_{j} \frac{\partial \widehat{u}_{i}}{\partial k_{l}}
$$


The first and the last terms in the right-hand side of the latter equation are collectively treated as a derivative along characteristic curves, which plays the same role as the mean trajectories in physical space. This derivative will, therefore, also be represented by a superimposed dot so that

$$
\dot{k}_{i}+U_{j, i} k_{j}=0, \quad \text { with solution } k_{i}=F_{j i}^{-1}(t, 0) K_{j} .
$$

The analogy with physical space is complete, since

$$
\dot{x}_{i}-U_{i, j} x_{j}=0, \quad \text { with solution } x_{i}=F_{i j}(t, 0) X_{j} .
$$

The initial $\mathbf{k}$ value, $K$, plays the same role in wave space as the Lagrangian coordinate $\mathbf{X}$ does in physical space. Pure kinematic distortion by advection in physical and spectral space are linked by a wave conservation law

$$
\exp \left(\mathrm{i} k_{j} x_{j}\right)=\exp \left(\mathrm{i} K_{j} X_{j}\right)
$$

where $\mathrm{i}^{2}=-1$. Accordingly, one has

$$
\widehat{\mathcal{F}}\left(\dot{u}_{i}\right)=\dot{\widehat{u}}_{i}-U_{1, l} \widehat{u}_{i}
$$

and equation (16) becomes

$$
\dot{\hat{u}}_{i}-U_{l, l} \widehat{u}_{i}+U_{i, j} \widehat{u}_{j}=-\mathrm{i} k_{i} \frac{\hat{p}}{\bar{\rho}} .
$$

In the latter equation, the projection operators in (19) can be used to separate solenoidal and dilatational contributions. We prefer to use a slightly different method by specifying a special frame for the solenoidal mode, according to an extended Craya-Herring decomposition (Cambon 1990). An orthonormal frame of reference $\left(\mathrm{e}^{(1)}, \mathrm{e}^{(2)}, \mathrm{e}^{(3)}\right)$ attached to the wavevector is used with the last vector being parallel to $\mathbf{k}\left(e_{i}^{(3)}=k_{i} / k\right.$, where $\mathrm{k}$ is the wavevector modulus). In this local frame, the Fourier transform of the velocity fluctuation reads

$$
\widehat{u}_{i}(\mathbf{k}, t)=\widehat{\varphi}^{(1)}(\mathbf{k}, t) e_{i}^{(1)}(\mathbf{k})+\widehat{\varphi}^{(2)}(\mathbf{k}, t) e_{i}^{(2)}(\mathbf{k})+\widehat{\varphi}^{(3)}(\mathbf{k}, t) e_{i}^{(3)}(\mathbf{k})
$$

The two first terms give exactly $\widehat{u}_{i}^{s}$, and the latter gives $\widehat{u}_{i}^{d}$, with a minimal number of components and conservation of all the tensorial properties (invariants) due to the orthonormal properties of the local frame. Classic descriptions in terms of vorticity and divergence are easily recovered as

$$
\widehat{\omega}_{i}=i k\left(\widehat{\varphi}^{(1)} e_{i}^{(2)}-\hat{\varphi}^{(2)} e_{i}^{(1)}\right)
$$

and

$$
\widehat{d} \equiv \widehat{u}_{i, i}=i k \widehat{\varphi}^{(3)}
$$


In order to remove the uncertainty regarding the azimuthal position of the solenoidal coordinates with respect to $\mathrm{e}^{(3)}$, the $\left(\mathrm{e}^{(1)}, \mathrm{e}^{(2)}\right)$ plane is defined by choosing a fixed spherical coordinate polar axis $n$, after Herring (1974). (Craya(1958) implicitly used $n_{i}=\delta_{i 3}$ and addressed only covariance matrices of the velocity field and thus limited the generality of his approach.) We set

$$
e^{(1)}=\frac{k \times n}{|k \times n|} \text { and } e^{(2)}=e^{(3)} \times e^{(1)} .
$$

Striking simplifications can be made by choosing the polar axis according to the symmetries of the mean flow (if any) or the statistical properties of the fluctuating field, retaining the full generality of the method. The equations in the local frame can be made nearly independent of the choice of $\mathbf{n}$ by using the "helical modes" $\left(e_{i}^{(2)}-\mathrm{i} e_{i}^{(1)}, e_{i}^{(2)}+\mathrm{i} e_{i}^{(2)}\right.$ ) (which are also eigenmodes of the plane rotation matrix around $k$ and of the "curl" operator) as the basis set (see Greenspan 1968, Cambon \& Jacquin 1989, Waleffe 1992). Substituting (24) into (23) leads to the linear system of equations for the three components of $\widehat{u}_{i}$ in the local Craya-Herring frame, with:

$$
\begin{gathered}
\dot{\hat{\varphi}}^{(\alpha)}-U_{l, l} \hat{\varphi}^{(\alpha)}+m_{\alpha \beta} \widehat{\varphi}^{(\beta)}+m_{\alpha 3} \widehat{\varphi}^{(3)}=0 \\
\dot{\hat{\varphi}}^{(3)}-U_{l, l} \widehat{\varphi}^{(3)}+m_{33} \hat{\varphi}^{(3)}+m_{3 \alpha} \widehat{\varphi}^{(\alpha)}+\mathrm{i} k \frac{\widehat{p}}{\bar{\rho}}=0 .
\end{gathered}
$$

Greek indices (indicating solenoidal space) take only the value 1 or 2 , whereas the Latin indices range from 1 to 3 (and as in physical space, the Einstein summation convention is assumed). Calculation of the matrix $m_{i j}$ is straightforward; remembering to account for the rotation due to the time derivative of the local frame at fixed $\mathrm{K}$ using eqs. (20) and (26), the elements are

$$
\begin{gathered}
m_{\alpha \beta}=e_{i}^{(\alpha)} U_{i, j} e_{j}^{(\beta)}-\dot{e}_{i}^{(\alpha)} e_{i}^{(\beta)}=e_{i}^{(\alpha)} U_{i, j} e_{j}^{(\beta)}-\epsilon_{\alpha \beta 3} R_{E} \\
m_{\alpha 3}=e_{i}^{(\alpha)} U_{i, j} e_{j}^{(3)}-\dot{e}_{i}^{(\alpha)} e_{i}^{(3)}=e_{i}^{(\alpha)}\left(U_{i, j}-U_{j, i}\right) e_{j}^{(3)} \\
m_{3 \alpha}=e_{i}^{(3)} U_{i, j} e_{j}^{(\alpha)}-\dot{e}_{i}^{(3)} e_{i}^{(\alpha)}=2 e_{i}^{(3)} U_{i, j} e_{j}^{(\alpha)} \\
m_{33}=e_{i}^{(3)} U_{i, j} e_{j}^{(3)}
\end{gathered}
$$

The rotation term $R_{E}$ is $e_{i}^{(2)} U_{i, j} e_{j}^{(1)}$ if the polar axis is chosen as one of the eigenvectors of the mean gradient matrix; its general expression is available in Cambon et al. (1985). The last equation relevant to our study is that which governs the pressure:

$$
\left(\frac{\dot{\hat{p}}}{\gamma P}\right)-U_{l, l}\left(\frac{\hat{p}}{\gamma P}\right)=-\widehat{d}=-\mathrm{i} k \hat{\varphi}^{(3)}
$$

Without mean distortion, eqs. (28) and (30) correspond to a pure acoustic regime, where energy is exchanged between dilatational velocity and pressure at a frequency 
$a k$. (The sonic speed $a$ is easily reintroduced using the isentropic relation $a^{2}=$ $\gamma R T=\gamma P / \bar{\rho}$.) On the other hand, the (exact) balance between the two last terms in Eq. (28) is the equivalent in physical space of the Poisson equation for $p$ in the pure solenoidal flow. The solenoidal contribution to velocity is seen to be completely uncoupled from the dilatational field if $m_{\alpha 3}$ is zero. This is valid for any irrotational compressing mean flow, but not for pure shear, as has been stressed by BMR. Finally, we note that the coupling of the solenoidal and dilatational fields is mediated by $m_{3 a}$. This term is zero for spherical compression but must be considered for any anisotropic straining process (except for very specific wavevectors given by the particular deformation). An investigation of the timescales in (28) introduces the parameter $R_{a}(k) \equiv\left(\tau_{D}\right)^{-1} / a k$, for which $\Delta m$ is an averaged approximation in physical space. For very low values of this parameter, the incompressible limit is recovered, the dilatational mode $\varphi^{(3)}$ tends to zero, and the sonic speed $a$ approaches infinity; both $k \hat{p} / \bar{\rho}$ (which tends to the solenoidal solution to the Poisson equation) and its time derivative (which from (30) is observed to be proportional to $a^{2} \varphi^{(3)}$ ) tend to finite non-zero values without inconsistency. At moderate $R_{a}(k)$, a pseudoacoustic regime is recovered, which deviates from the pure incompressible $\left(u_{i, i}=0\right.$, $p=p_{s}$ ) case since the time variation of the $m_{3 \alpha}$ term in (28) can be neglected and a WKB approximation can be used to predict the oscillating behavior of $\hat{d}$ (Sabel'nikov 1975 and DZ). (This oscillating behavior will be revisited in §5.) For large values of $R_{a}(k)$, the pressure term in Eq. (28) can be neglected compared to the other terms, and the "pressure released" regime is obtained. We note that use of the solenoidal Poisson equation to approximate the total pressure variance (i.e. setting $p=p_{s}$ ) and then using (30) to estimate the pressure-dilatation term, a method followed by $\mathrm{DZ}$, can lead to some inconsistencies. If $p=p^{*}$ holds, the dilatational mode is directly given by a simplification of (28) (equating the first three terms to zero), and the solution is

$$
\widehat{\varphi}^{(3)}(\mathbf{k}, t)=J \frac{k}{K} \widehat{\varphi}^{(3)}(\mathbf{K}, 0)
$$

The potential inconsistency is that this solution for $q_{d}^{2}$ is not necessarily the same as that found from Eq. (30). In (31), the dilatational part of the kinetic energy depends only upon its initial value; for a mean compression, $q_{d}^{2}(t)=\mathcal{F}(J(t)) q_{d}^{2}(0)$, where $\mathcal{F}(J)$ depends on the type of compression. In contrast, the $\mathrm{DZ}$ method amounts to connecting both $\hat{p}$ and $\hat{\varphi}^{(3)}$ to the initial value of the solenoidal modes, $\hat{\varphi}^{(\alpha)}(\mathbf{K}, 0)$, so that the dilatational part of the kinetic energy depends only on the solenoidal initial data: $q_{d}^{2}(t)=\mathcal{F}_{\mathrm{DZ}}(J(t))(\Delta m)^{4} q_{s}^{2}(0)$ (where $\mathcal{F}_{\mathrm{DZ}}(J) \neq \mathcal{F}(J)$ and again depends upon the compression type).

An approach which avoids this ambiguity and allows the classification of other relevant limits is available by introducing integrating factors into (28) and (30) so that

$$
y=J^{-1} \frac{\hat{\varphi}^{(3)}}{k} \quad \text { and } \quad z=J^{-1} \frac{\hat{p}}{\bar{\rho} a^{2}}
$$




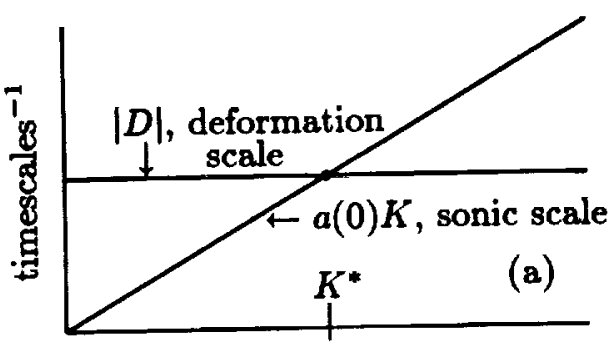

K

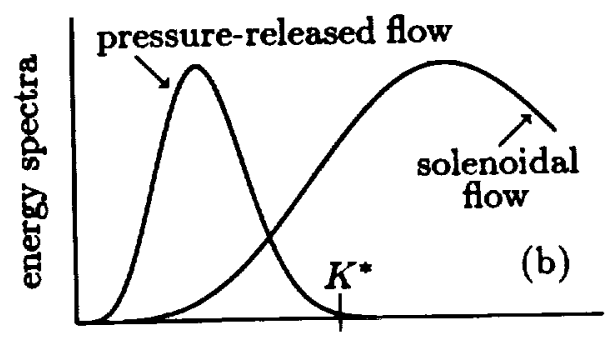

K

FigurE 1. Solenoidal and pressure-release regimes.

satisfy the simpler equations:

$$
\begin{gathered}
\frac{\mathcal{D}\left(\dot{y} / a^{2}\right)}{\mathcal{D} t}+k^{2} y=\mathrm{i} z^{*} \\
\frac{\mathcal{D}\left(\dot{z} / k^{2}\right)}{\mathcal{D} t}+a^{2} z=a^{2} z^{s},
\end{gathered}
$$

where $z^{s}=J^{-1} \widehat{p}_{s} / \bar{\rho} a^{2}=\mathrm{i}\left(J^{-1} / k a^{2}\right) m_{3 \alpha} \hat{\varphi}^{(\alpha)}$. The left-hand sides of both (33) and (34) are linked only to the dynamics of the solenoidal field and thus decoupled from the dilatational and pressure terms for irrotational mean deformations.

We are now in a position to distinguish the different regimes implied by equations (33) and (34):

I. The incompressible limit, with $a^{2} \rightarrow \infty$, which corresponds to a vanishing value of all the time-derivatives in both equations; hence, $z^{b}$ and $y \rightarrow 0$, and $z=z^{s}$ (i.e. $p=p_{s}$ ) and $y=0$ are consistent limits in this case.

II. The acoustic regime, recovered when $k^{2} y \gg \mathrm{i} z^{3}$.

III. The regime studied by Durbin \& Zeman, where the pressure-dilatation correlation is given by the solenoidal pressure variance, which corresponds to $k^{2} y=\mathrm{i} z$ in (33) and $z=z^{s}$ in (34); these equalities hold only if the time-derivative of the solenoidal term (right-hand side of (33)) is much larger than the time-derivative of the dilatational term (first term on the left-hand side of (33)).

IV. The pressure-released limit, corresponding to $k^{2} y \ll \mathrm{i} z$ in (33), which leads to the condition

$$
\overline{u_{i, i}^{2}} \ll(\Delta m)^{4} \frac{q_{s}^{2}}{L^{2}}
$$

(with $L$ a lengthscale of the energy containing turbulence) required for the pressure-released regime to be valid. We mention in passing that if one assumes that the ratio $\lambda$ of the dilatational to solenoidal kinetic energy is proportional to $M_{t}^{2}$, the above inequality suggests that an alternative to $\Delta m$ as the parameter that defines the pressure-released regime is the quantity $\Delta m M_{t}^{-1 / 2}=r M_{t}^{1 / 2}$. In spite of this, the DNS results presented below indicate that the pressure-released limit seems to be adequately parameterized by $\Delta m$ alone. 
Complete solutions of the system of linear equations (28) and (30) are required for intermediate values of $R_{a}(k)$. The method described in Cambon (1982) of extending the Townsend (1976) approach can also be used for the general (homogeneous) case; an overview of the scheme follows, which we plan to follow in future work. A linear transfer function $\mathbf{g}$, which generates the general solution of the system of equations as

$$
\widehat{\varphi}^{(i)}(\mathbf{k}, t)=g_{i j}\left(\mathrm{e}^{(3)}, K / K_{0}, R_{a}\left(K_{0}\right), a_{0} K_{0} t\right) \hat{\varphi}^{(j)}(\mathbf{K}, 0),
$$

is computed by solving (numerically in general) (20), (27), (28), and (30) for a set of arbitrary simple initial data $\left(\hat{\varphi}_{0}^{(i)}=\delta_{i 1},=\delta_{i 2}\right.$, etc...). (For convenience, the subscript can be taken to vary from 1 to 4 in order to represent the pressure fluctuation with $\widehat{\varphi}^{(4)}=\hat{p} / \bar{\rho} a$.) All the relevant one-point correlations can then be obtained by integrating over spectral space products of linear transfer functions and initial spectra. The initial spectra such as

$$
\left\langle\widehat{\varphi}^{(i) \star}(\mathbf{P}, 0) \hat{\varphi}^{(j)}(\mathbf{K}, 0)\right\rangle=\frac{E^{(i j)}(K)}{4 \pi K^{2}} \delta(\mathbf{K}-\mathbf{P})
$$

can be generated by invoking isotropy and assuming acoustic equilibrium (Sarkar et al. 1989) and certain relationships between the dilatational spectra (Bataille, et al. 1992). Note that the delta function $\delta$ amounts to a factor $\delta_{n m}(\Lambda(0) / 2 \pi)^{3}$ if discrete Fourier modes $(P=n, K=m)$ are chosen using periodic boxes of size $\Lambda$, as is done in DNS. For both the continuous and discrete case, the mean compression must be taken into account when computing correlations and integrating over wave space, using either

$$
\delta(\mathbf{k}-\mathbf{p})=\delta(\mathbf{K}-\mathbf{P}) J(t)
$$

or $\Lambda^{3}(t) / \Lambda^{3}(0)=J$ and $d^{3} \mathbf{k}=d k_{1} d k_{2} d k_{3}=J^{-1} d^{3} \mathbf{K}$. For the solenoidal field certain results can be obtained analytically, as is demonstrated below for the case of axial compression, since $g_{i j}^{s}$ depends only on the orientation of the wave vector, and not on the modulus; integrations over wave space needed to derive the velocity correlations can thus be separated into the product of two one-dimensional integrals, one of which defines (independently of initial spectra shape) the initial kinetic energy. Evaluation of the non-solenoidal correlations is not as straightforward since the components of the linear transfer matrix that involve the dilatation depend on both the direction $e_{i}^{(3)}=k_{i} / k$ of the wavevector $\mathrm{k}$ (as for the solenoidal case) and on its modulus. Accordingly, amplification coefficients like the functions $\mathcal{F}$ and $\mathcal{F}_{D Z}$ mentioned above in general require numerical integration. This complication is a symptom of the wave number dependence of the sonic timescale in spectral space $(a(0) K)^{-1}$, symbolically shown in Figure 1a; since the deformation scale $D^{-1}$ is the same for all wave numbers, above a critical value $K^{*}$, the sonic is the shorter of the two timescales. For a given energy spectrum with peak at $K_{0}$ so that $\Delta m$ is characterized by $R_{a}\left(K_{0}\right)$, the rapid distortion behavior depends on $K / K_{0}$. The largest structures $\left(K<K_{0}\right)$ will, therefore, naturally tend toward the pressure-released extreme and the smallest $\left(K>K_{0}\right)$ toward the solenoidal limit. 

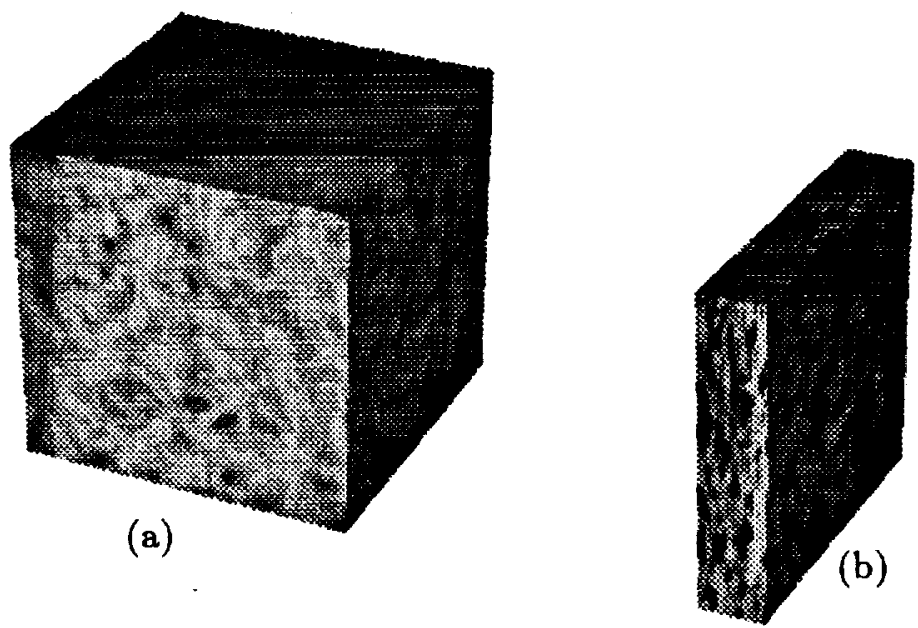

FIGURE 2. Contours of turbulent Mach number (a) before and (b) after axial compression (Case C1DW).

When $K_{0}$ falls well below $K^{*}$, the entire flow is within the pressure-released regime, and $\Delta m \gg 1$; when $K_{0} \gg K^{*}$, the $\Delta m \rightarrow 0$ limit is valid (see Figure $1 \mathrm{~b}$ ).

In the next section, the analysis is applied to the special case of axial compression, and DNS results are used to verify the relevance of $\Delta m$ as a critical parameter.

\section{RDT and DNS of axially compressed flow}

Both the RDT and DNS impose upon isotropic compressible turbulence the axial deformation that satisfies the homogeneity condition (15) so that the single nonzero mean velocity gradient component is

$$
U_{1,1}=\frac{D_{0}}{1+D_{0} t}=D_{0} J^{-1} \text { and } F_{11}=J
$$

For $D_{0} \equiv D(0)<0$, this straining can be maintained for a finite time for as long as the flow volume is nonzero. Here we consider mean density ratios (equal to $J^{-1}$ ) that vary from 1 to 5 ; see Figure 2. Before describing the various DNS runs, which is done in $\S \S 3.2$, in the next subsection we specify the RDT correlations relevant to one point modeling of the axial compression.

\subsection{Rapid distortion analysis for axial compression}

For the case of axial compression, the Craya-Herring-Cambon coordinates given in $\S 2$ reduce to $e_{1}^{(3)}=\cos \theta, e_{1}^{(2)}=-\sin \theta$, where $\theta=(\mathbf{k}, \mathbf{n})$ if the polar axis is chosen along the compression direction so that $e_{1}^{(1)}=0$ (see Cambon \& Jacquin (1989) for other axisymmetric RDT applications). The RDT solutions for the solenoidal field are then

$$
\hat{\varphi}^{(1)}(\mathbf{k}, t)=J \hat{\varphi}^{(1)}(\mathbf{K}, 0) \quad \text { and } \quad \hat{\varphi}^{(2)}(\mathbf{k}, t)=\frac{K}{k} \widehat{\varphi}^{(2)}(\mathbf{K}, 0),
$$


with $k_{1}=K_{1} J^{-1}, k_{2}=K_{2}, k_{3}=K_{3}, \cos \theta=k_{1} / k=\xi J^{-1} /\left(1+C^{2} \xi^{2}\right)^{1 / 2}, k^{2}=$ $1+C^{2} \xi^{2}, C^{2}=J^{-1}-1$ and $\xi=K_{1} / K$. The double correlations are calculated using

$$
\left\langle\widehat{u}_{i}^{\star} \widehat{u}_{i}\right\rangle=\left\langle\widehat{\varphi}^{(i) \star} \hat{\varphi}^{(i)}\right\rangle \text { and } \widehat{u}_{1}=-\widehat{\varphi}^{(2)} \sin \theta+\widehat{\varphi}^{(3)} \cos \theta
$$

Assuming isotropic initial data, both the solenoidal and pressure-released analytical RDT predictions can be obtained by integrating either over $\xi$ or directly in physical space, with the results being unaffected by the initial spectral shape. The axial compression correlations are tabulated below, using as super- or subscripts " $s$ " and " $p$ ", respectively, to denote the solenoidal and pressure-released limiting cases.

Turbulent kinetic energy:

$$
\begin{aligned}
& \frac{q_{s}^{2}(t)}{q_{s}^{2}(0)}=A_{s}(J), \\
& \frac{q^{2}(t)}{q^{2}(0)}=A_{p}(J) .
\end{aligned}
$$

Compression-direction Reynold stress component:

$$
\begin{aligned}
& \frac{\left\langle u_{1}^{s} u_{1}^{s}\right\rangle(t)}{q_{a}^{2}(0)}=B_{a}(J), \\
& \frac{\left\langle u_{1} u_{1}\right\rangle(t)}{q^{2}(0)}=B_{p}(J) .
\end{aligned}
$$

Compression-direction solenoidal-dilatational cross-correlation:

$$
\begin{gathered}
\left\langle u_{1}^{s} u_{1}^{d}\right\rangle(t)=0, \\
\frac{\left\langle u_{1}^{s} u_{1}^{d}\right\rangle(t)}{q_{s}^{2}(0)}=C_{p}(J) .
\end{gathered}
$$

Structure dimensionality tensor (Reynolds 1990; see also (47) and (48)):

$$
\begin{gathered}
\frac{D_{11}^{s}(t)}{q_{s}^{2}(0)}=D_{s}(J)=B_{s}(J), \\
\frac{D_{11}(t)}{q^{2}(0)}=D_{p}(J) .
\end{gathered}
$$

Compression-direction component of the pressure-strain rate correlation:

$$
\begin{aligned}
& \frac{\Pi_{11}^{s}(t)}{q_{s}^{2}(0)}=D E_{s}(J), \\
& \Pi_{11}=E_{p}(J)=0 .
\end{aligned}
$$


The pressure-dilatation term $\Pi$ is identically zero for both limits. In terms of the inverse compression ratio $J$ with $C^{2} \equiv J^{-2}-1$, we have for the $J<1$ case:

$$
\begin{gathered}
A_{s}=\frac{1}{2}\left(1+J^{-2} \frac{\tan ^{-1} C}{C}\right) \\
A_{p}=\frac{2+J^{-2}}{3} \\
B_{s}=\frac{J^{-2}}{2 C^{2}}\left(\frac{1}{2}+\frac{C^{2}-1}{2} \frac{\tan ^{-1} C}{C}\right) \\
C_{p}=\frac{J^{-2}}{2 C^{2}}\left(-1+J^{-2} \frac{\tan ^{-1} C}{C}\right)-B \\
D_{p}=\frac{J^{-2}}{C^{2}}\left(1-\frac{\tan ^{-1} C}{C}\right)\left(\frac{2+J^{-2}}{3}\right) \\
E_{s}=\frac{1}{2 C^{4}}\left(C^{2}+3+\left(C^{2}-3\right) J^{-2} \frac{\tan ^{-1} C}{C}\right) .
\end{gathered}
$$

The solenoidal amplification functions turn out to be nearly linear in $J^{-1}$, whereas the pressure-released expressions are nearly parabolic. The quantities $A_{a}$ and $B_{a}$, previously derived by Ribner(1953), and the new expressions $D_{s}$ and $E_{s}$ are almost the same as those found for incompressible axisymmetric strain (of arbitrary history), with, for example, the Reynolds stress tensor $R_{i j}^{s}=\left\langle u_{i}^{s} u_{j}^{s}\right\rangle=J^{-2 / 3} R_{i j}^{*}(J)$, where $\mathbf{R}^{*}$ is the RDT solution for the trace-free part of the mean deformation. Functions $A_{p}$ and $B_{p}$ are obtained by simply ignoring the pressure terms during the integration of the equations for the one-point correlations in the rapid axial compression limit, which are:

$$
\begin{gathered}
\frac{1}{2} \dot{q}^{2}=-D R_{11}+\Pi, \\
\dot{R}_{11}=-2 D R_{11}+\Pi_{11},
\end{gathered}
$$

where $R_{i j}=\left\langle u_{i} u_{j}\right\rangle$, and $\Pi=\Pi_{i i} / 2=\left\langle p u_{i, i}\right\rangle / \bar{\rho}$.

For moderate compressibility, we find from the DNS results that the role of $\Pi_{11}$, which reduces the anisotropy $\left(b_{11}=R_{11} / q^{2}-1 / 3\right)$ in (44) and, therefore, indirectly reduces the production term in the kinetic energy equation, is more important than the direct role of $\Pi$. For future reference, we now put forth some useful results concerning the anisotropy tensor $b_{i j}=\left\langle u_{i} u_{j}\right\rangle / q^{2}-\frac{1}{3} \delta_{i j}$, and a recommended general decomposition for the Reynolds stress:

$$
R_{i j}=q_{s}^{2}\left(\frac{\delta_{i j}}{3}+b_{i j}^{e(s)}+b_{i j}^{z(s)}\right)+\left\langle u_{i}^{s} u_{j}^{d}\right\rangle+\left\langle u_{i}^{d} u_{j}^{s}\right\rangle+q_{d}^{2}\left(\frac{\delta_{i j}}{3}+b_{i j}^{e(d)}\right)
$$


where the solenoidal deviator $b_{i j}^{g}$ is split into a part reflecting the directional dependence (superscript $e$ ) and the "polarization" (subscript $z$ ) (Cambon et al. 1992). This decomposition can be recovered using the "structure tensors" introduced by Reynolds(1990) (see also BMR) via

$$
\begin{aligned}
& D_{i j}^{s}=\left\langle\psi_{l, i} \psi_{l, j}\right\rangle=\int 2 \frac{k_{i} k_{j}}{k^{2}} e^{s}(\mathbf{k}, t) d^{3} \mathbf{k}=q_{o}^{2}\left(\frac{\delta_{i j}}{3}-2 b_{i j}^{e(s)}\right) \\
& C_{i j}^{s}=\left\langle\psi_{i, l} \psi_{j, l}\right\rangle=\int \frac{e_{i j}^{w}(\mathbf{k}, t)}{k^{2}} d^{3} \mathbf{k}=q_{s}^{2}\left(\frac{\delta_{i j}}{3}+b_{i j}^{e(s)}-b_{i j}^{z(s)}\right) \\
& D_{i j}^{d}=\left\langle\varphi_{, i} \varphi_{, j}\right\rangle=\int 2 \frac{k_{i} k_{j}}{k^{2}} e^{d}(\mathbf{k}, t) d^{3} \mathbf{k}=q_{d}^{2}\left(\frac{\delta_{i j}}{3}+b_{i j}^{e(d)}\right) .
\end{aligned}
$$

To show the equivalence of the two approaches we first note that the tensors in (47) and (48) are formally defined using the vector $\psi_{i}$ or scalar $\varphi$ potential functions according to the Helmholtz decomposition (18) in physical space. The threedimensional spectra are then defined (the second equality in (47) and (48)), with $e^{s}$, $e^{d}$ and $e_{i j}^{\omega}$ being associated with respectively $\frac{1}{2}\left\langle\widehat{u}_{i}^{{ }^{\star}} \widehat{u}_{i}^{s}\right\rangle=\frac{1}{2}\left\langle\widehat{\varphi}^{(\alpha) \star} \widehat{\varphi}^{(\alpha)}\right\rangle, \frac{1}{2}\left\langle\widehat{u}_{i}^{d \star} \widehat{u}_{i}^{d}\right\rangle=$ $\frac{1}{2}\left\langle\widehat{\varphi}^{(3) \star} \widehat{\varphi}^{(3)}\right\rangle$, and $\left\langle\widehat{\omega}_{i}^{\star} \widehat{\omega}_{j}\right\rangle$. Finally, the directional-polarization anisotropy tensors are specified (the third equality) so that the two approaches are reconciled.

In the notation of Reynolds (1990), $D_{i j}^{s}$ reflects the "dimensionality" of the solenoidal field, which is close to the directional anisotropy, whereas $C_{i j}^{s}$ is associated with the "componentality" of the turbulence. Only the dimensionality, or directional anisotropy, is needed for the dilatational velocity field since $D_{i j}^{d}=\left\langle u_{i}^{d} u_{j}^{d}\right\rangle$ (BMR). This reflects the single-component character of $u_{i}^{d}$, in contrast to the twocomponent structure of the solenoidal field. We finally note that the above tensors are not all independent; for example, $C_{i j}^{s}$ can be derived from the other tensors using (46)-(48), or equivalently the equation found by Reynolds (1992):

$$
\left\langle u_{i}^{s} u_{j}^{s}\right\rangle+D_{i j}^{s}+C_{i j}^{s}=q_{s}^{2} \delta_{i j}
$$

It is hoped that the above general expressions will be useful in future attempts to model compressible flows. For the present, however, we narrow our approach as we use DNS results to test a few aspects of the rapid distortion analysis.

\subsection{Comparison to DNS of rapid axial compression}

The DNS results were obtained using a pseudo-spectral method to solve the compressible Navier-Stokes equations over a homogeneous domain in coordinates that move with the mean deformation (Rogallo 1981, BMR, Coleman \& Mansour 1991). As mentioned previously, the mean density ratio, $J^{-1}=\bar{\rho}(t) / \bar{\rho}(0)$ varies from 1 to 5 during the compression. The runs use for initial conditions compressible isotropic turbulence at various turbulent Mach numbers that have evolved from velocity fields, with finite dilatational components, that are in near acoustic equilibrium; these initial fields are generated by running the code with no mean straining until they develop realistic triple-velocity correlations and dilatational energy for 


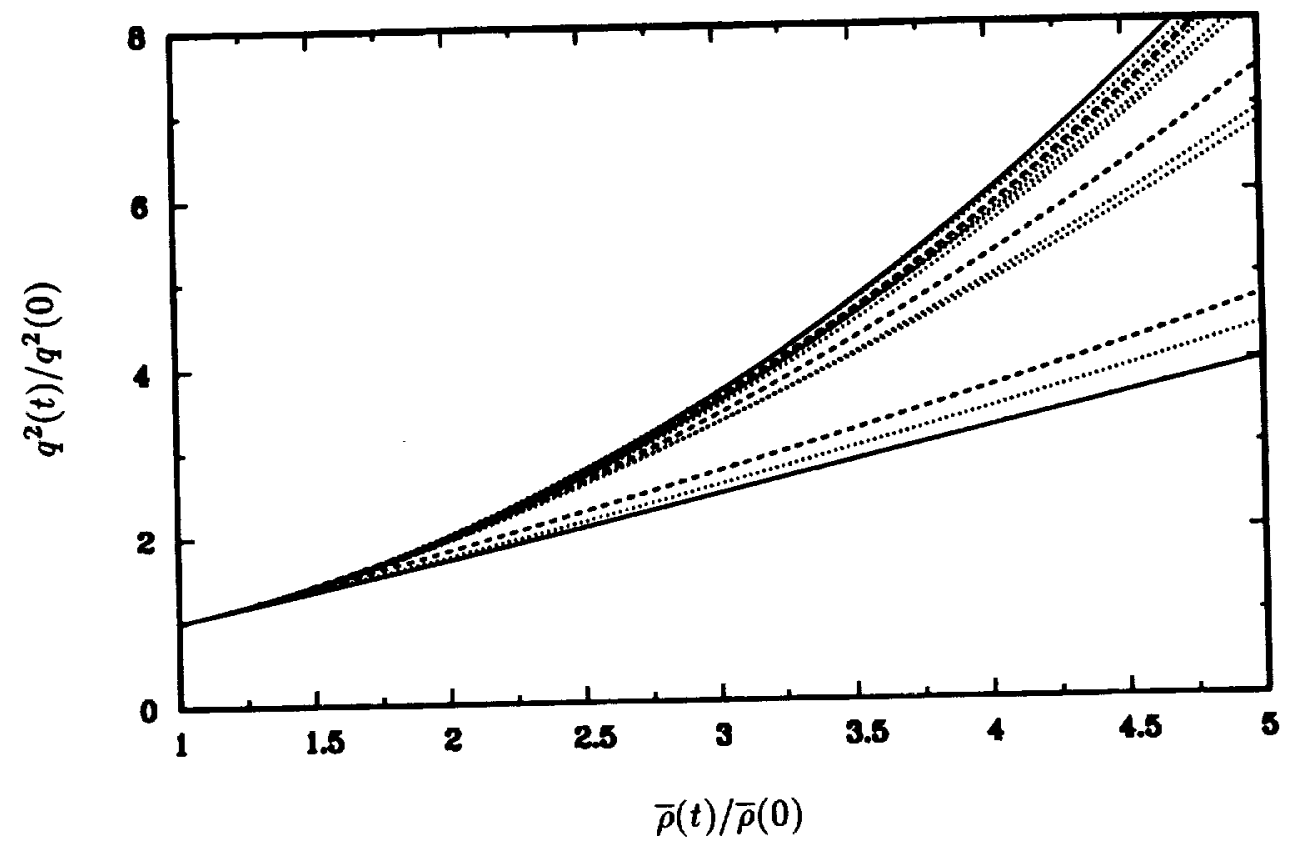

FIGURE 3. Turbulent kinetic energy histories: - , lower, Eq. (38a); upper, Eq. (38b); ….... DNS with initial $\Delta m$ ranging from 0.3 (lower) to from 3.0-8.0 (upper); ----, DNS Cases: lower, C1DJ $\left(M_{t}, \Delta m\right)_{t=0}=(0.03,0.3)$; upper, C1DV $(0.1,7)$; middle, C1DW $(0.3,1)$.

the given $M_{t}$. (Note that BMR have found that compressible isotropic turbulence strongly depends upon all the initial conditions for the dilatational field, not just $M_{t}$, which implies that had we begun the precomputation with, for example, a purely solenoidal field, the levels of dilatational energy in the developed flow might be significantly different than those found here.) The initial turbulent Mach number for the runs varies from 0.03 to 0.44 , the initial nondimensional compression speed $r=|D| q^{2} / \epsilon$ ranges from 50 to 800 (and $|D| /\left\langle\omega_{i} \omega_{i}\right\rangle^{1 / 2}$ from 2 to 88), and the initial values of $\Delta m=M_{t}|D| /\left\langle\omega_{i} \omega_{i}\right\rangle^{1 / 2}$ fall between 0.26 and 7 . A ratio of constant specific heats $\gamma=5 / 3$ and temperature dependent viscosity $\mu=T^{0.72}$ is assumed. All the runs used $96^{3}$ grid points and were generated on the Intel Hypercube/i860 at the NASA Ames Numerical Aerodynamic Simulation program.

Results for the total (solenoidal and dilatational) turbulent kinetic energy will first be presented. In Figure 3, the DNS histories for $\left\langle\rho u_{i} u_{i}\right\rangle / \bar{\rho}$ are plotted against the mean density ratio $J^{-1}=\bar{\rho}(t) / \bar{\rho}(0)$. (Because it is convenient in the code to solve for momentum rather than velocity, all of the DNS results presented approximate velocity correlations by using density weighted averages. We find for our purposes 


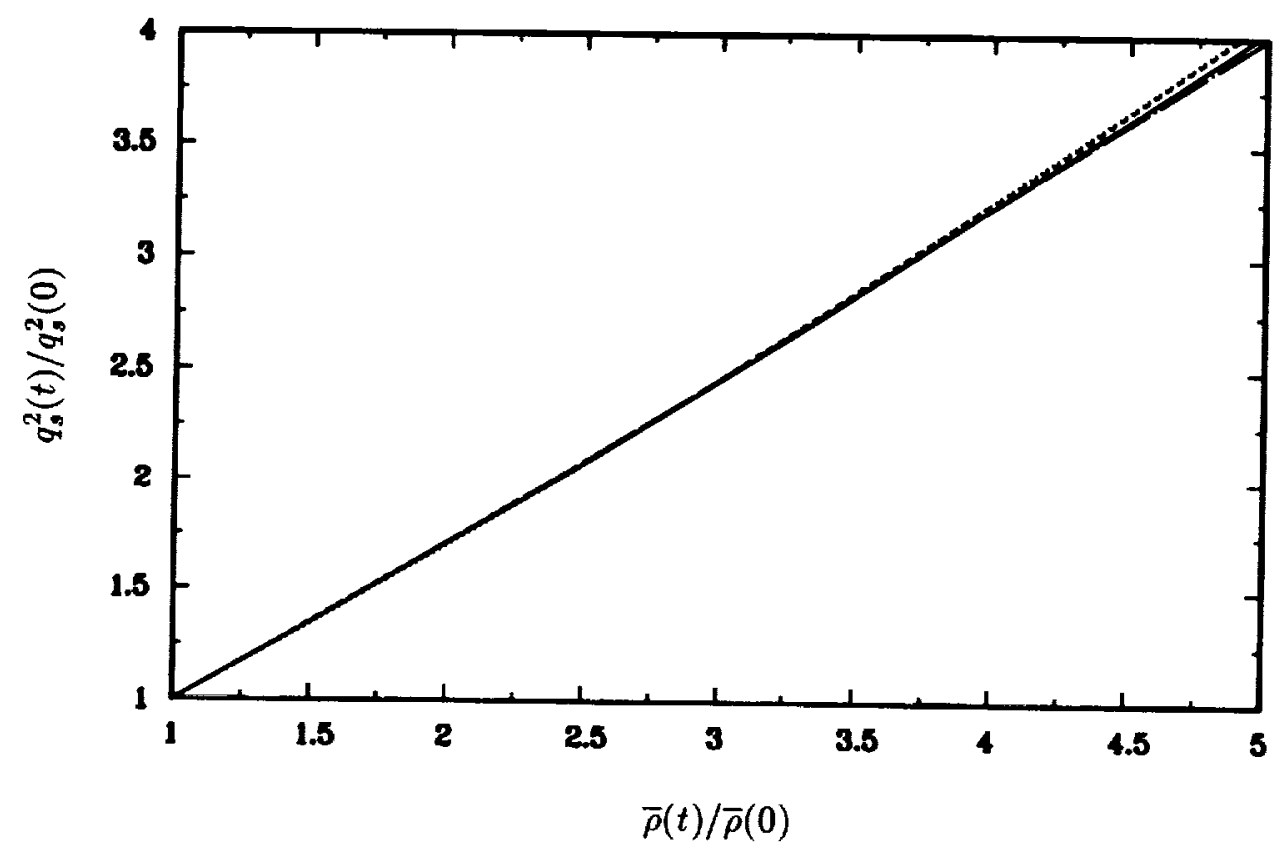

FIGURE 4. Solenoidal turbulent kinetic energy histories: $\Delta m)_{t=0}=(0.03,0.3) ;-\cdots, \operatorname{C1DV}(0.1,7) ; \cdots \cdots . ., \mathrm{C} 1 \mathrm{DW}(0.3,1) ;-.-$ Eq. $(38 a)$.

that the uncertainty introduced by comparing the DNS Favre averages to the RDT Reynolds averages is unimportant.) These curves strongly support the validity of the RDT analysis presented above in that all the DNS results lie between the lower solenoidal (" $A_{0}$ ") and upper pressure-releases (" $A_{p}$ ") RDT limits and that rate of energy amplification scales almost monotonically with the initial value of $\Delta m$, which varies from 0.3 for the lower (dotted) curve to from 3 to 8 for the upper (dotted) curves. Three runs will be examined further, those represented by the dashed curves in Figure 3. Cases C1DJ, C1DV, and C1DW have initial $M_{t}$ equal to $0.03,0.1$, and 0.3 , respectively, but the compression rates are such that the corresponding order for $\Delta m$ is $0.3,7$, and 1 . At the end of the compression the $\left(M_{t}, \Delta m\right)$ values for C1DJ, C1DV, and C1DW are respectively $(0.03,13),(0.2,79)$, and $(0.4,6)$.

Figure 4 confirms that Eq. (38a) is an excellent approximation for $q_{s}^{2}$ for the three cases considered and that the solenoidal field is, in fact, unaffected by the dilatational field, as predicted by the RDT. Both contributions to the kinetic energy are shown in Figure 5. We see that the dilatational energy is most important at the end of the compression, when the pressure-released regime dominates. The initial values of the dilatational-to-solenoidal energy ratio $\lambda_{0}$ for the various runs is also apparent. 


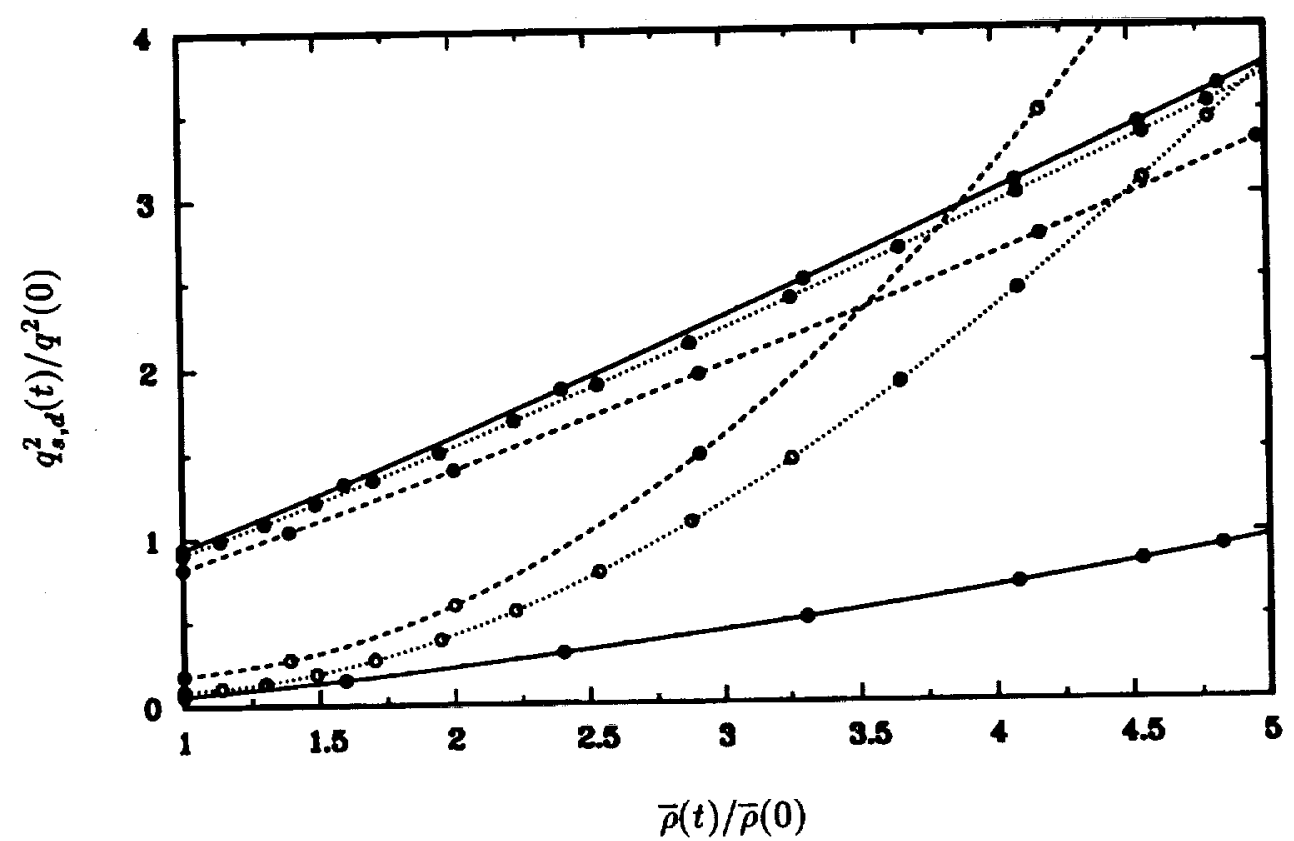

FIGURE 5. Solenoidal and dilatational turbulent kinetic energy histories: •, solenoidal; $O$, dilatational; $\longrightarrow, \operatorname{C1DJ}\left(M_{t}, \Delta m\right)_{t=0}=(0.03,0.3) ;-\cdots$, C1DV $(0.1,7) ; \cdots \cdots . ., \mathrm{C} 1 \mathrm{DW}(0.3,1)$.

These results suggest the following model for the Mach number dependence of the kinetic energy behavior during a rapid axial compression:

$$
\begin{gathered}
q_{s}^{2}(t)=A_{s}(J) q_{s}^{2}(0) \\
q_{d}^{2}(t)=A_{p}(J) q_{d}^{2}(0)+\left(A_{p}^{+}(J)-A_{s}^{+}(J)\right) q_{s}^{2}(0),
\end{gathered}
$$

where the "interpolation functions" $A_{p}^{+}$and $A_{s}^{+}$are assumed to vary monotonically with $\Delta m$, increasing from zero to maxima of $A_{p}$ and $A_{s}$, respectively. Similar agreement with DNS data is found for the other correlations given in (43). The results for $\left\langle u_{1}^{a} u_{1}^{p}\right\rangle$ and $\left\langle u_{1}^{d} u_{1}^{d}\right\rangle$ are presented on Figure 6 , where the DNS and RDT histories closely correspond. The slight overamplification of the DNS result compared to the analytical $\left\langle u_{1}^{s} u_{1}^{s}\right\rangle / q_{s}^{2}=B_{s} / A_{s}$ ratio becomes more pronounced with increasing $\Delta m$. For the dilatational curves in Figure $6,\left\langle u_{1}^{d} u_{1}^{d}\right\rangle / q_{d}^{2}$, we find the expected trend with $\Delta m$, since they are closest to the analytical pressure-released expression (the "chain-dash" curve) when $\Delta m$ is largest. An analog to (49) is therefore proposed as a model for the dilatational Reynolds stress:

$$
\frac{\left\langle u_{1}^{d} u_{1}^{d}\right\rangle}{q_{d}^{2}}=\frac{B_{p}(J) \lambda_{0}+B_{p}^{+}(J)-B_{s}^{+}(J)}{A_{p}(J) \lambda_{0}+A_{p}^{+}(J)-A_{s}^{+}(J)},
$$




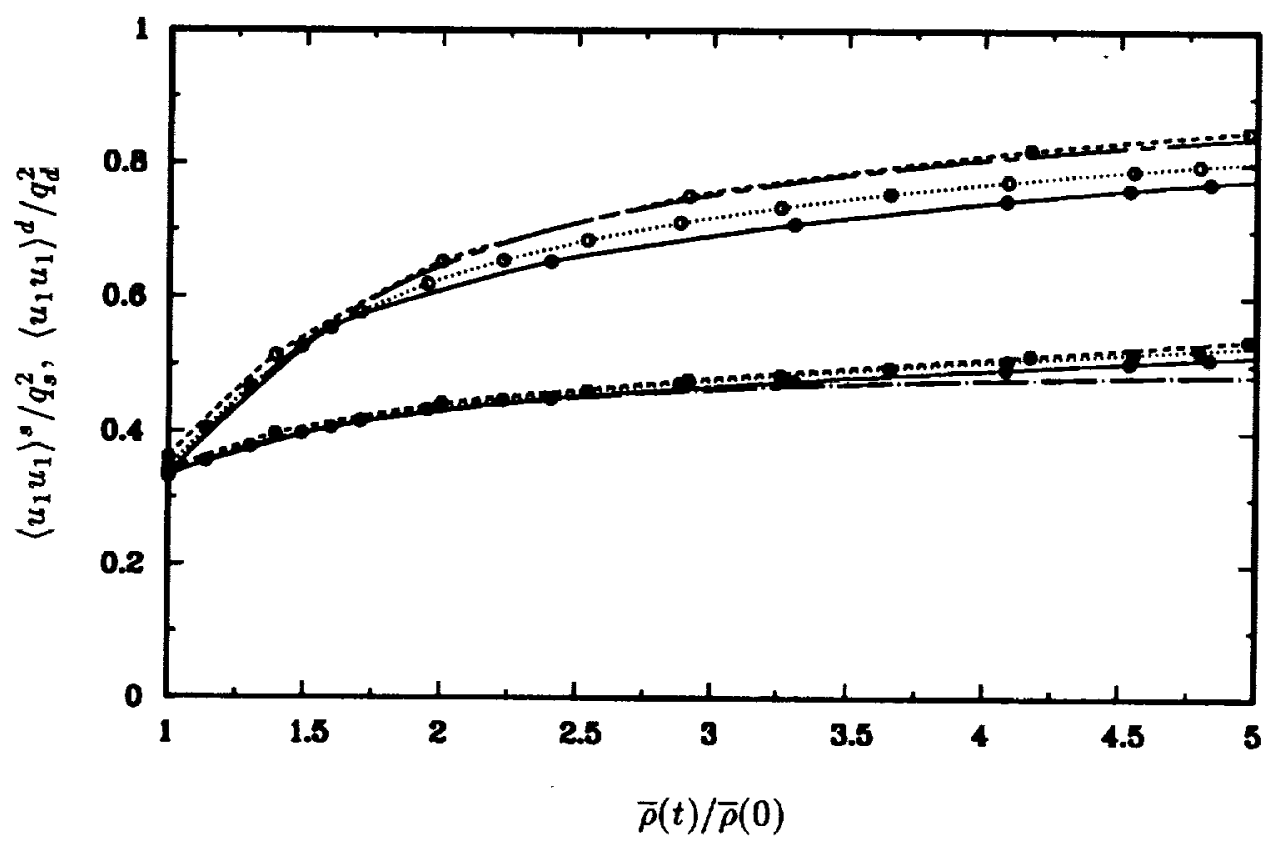

Figure 6. Histories of the anisotropy of solenoidal and dilatational turbulent kinetic energy: •, solenoidal; $O$, dilatational;,$- \operatorname{C1DJ}\left(M_{t}, \Delta m\right)_{t=0}=(0.03,0.3)$; ,--- C1DV $(0.1,7) ; \cdots \cdots . .$. C1DW $(0.3,1) ;-.-$, Eqs. $(38 a),(39 a) ;---$, Eqs. $(38 a, b),(39 a, b)$, using $\lambda_{0}=0.22$ from Run C1DV.

where $\lambda_{0}$ is the initial ratio of the dilatational to solenoidal kinetic energy (which in practice might be neglected). The curves in Figure 6 suggest that the ratio $\left(B_{p}^{+}-B_{s}^{+}\right) /\left(B_{p}-B_{s}\right)$ is smaller than the same ratio of " $A$ " functions.

Another anisotropy measure is investigated in Figure 7, where the structure tensors are presented. Recall that $D_{11}^{d}=\left\langle u_{1}^{d} u_{1}^{d}\right\rangle$. The fact that $D_{s}=B_{s}$ in (41a) confirms that $b_{i j}^{e(s)}=-\frac{1}{2} b_{i j}^{s}$ and $b_{i j}^{z(s)}=\frac{3}{2} b_{i j}^{s}$ is a good approximation for axisymmetric strain, as suggested by studies of non-isotropic initial data under rapid rotation (Reynolds 1990, Cambon et al. 1992, Mansour et al. 1991). Rapid rotation was shown to damp $b_{i j}^{z(s)}$ and, therefore, to reveal the initial anisotropy of $b_{i j}^{e(s)}$ as the asymptotic limit reached after several revolution times. In axisymmetric turbulence, $D_{11} / q^{2}$ can be interpreted as an angular coefficient $\cos ^{2} \alpha$, as implied by the integrands in (47) and (48), which reveals the conical structure of the spectral region that contains energy (around the symmetry axis). For example, a value of $1 / 3$ for this coefficient suggests no angular dependence (directional isotropy), whereas a value between 0 and $1 / 3$ suggests a relative concentration of spectral energy in the plane normal to the symmetry axis. Unfortunately, the situation is 


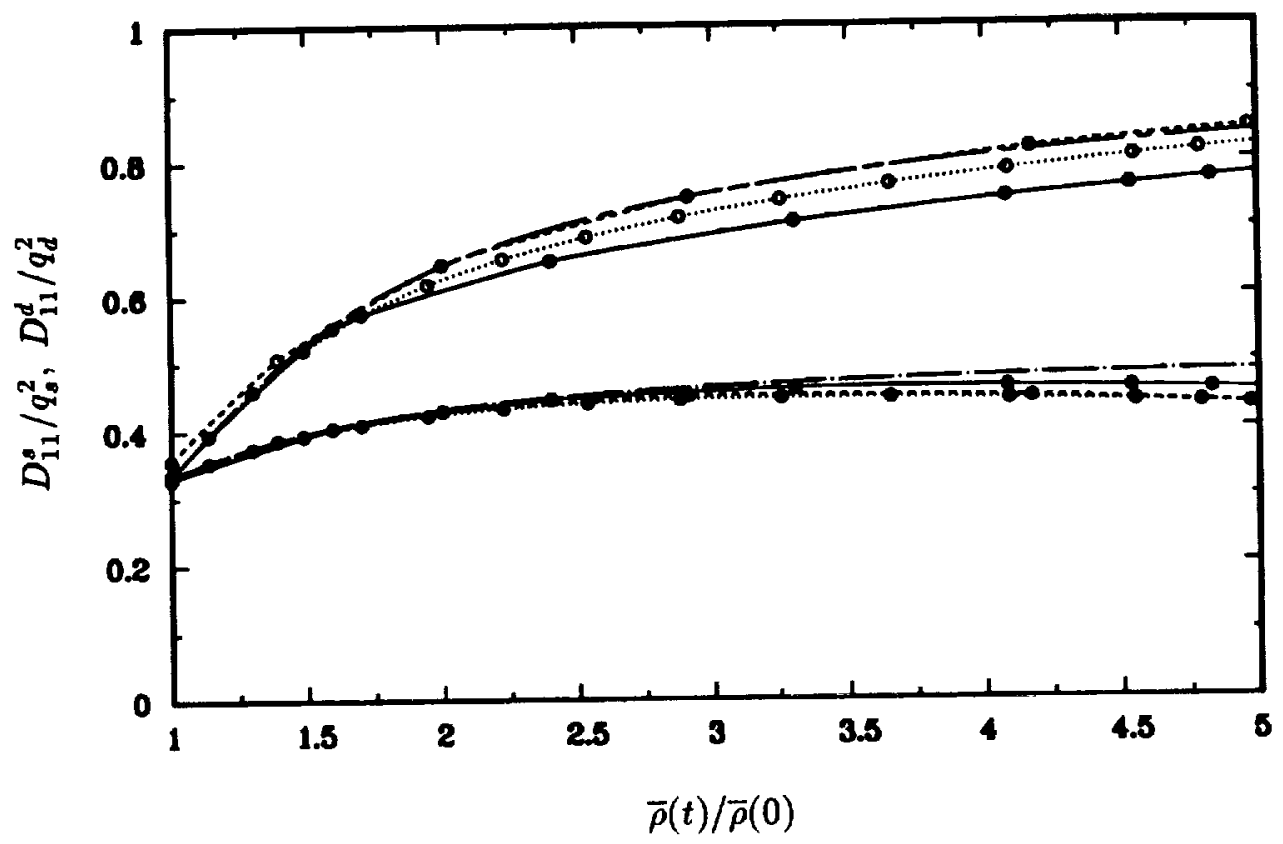

FIGURE 7. Structure tensor histories: $\bullet$, solenoidal; 0 , dilatational; - , C1DJ $\left(M_{t}, \Delta m\right)_{t=0}=(0.03,0.3) ;-\cdots$, C1DV $(0.1,7) ; \cdots \cdots . . .$, C1DW $(0.3,1) ;-.-$, Eqs. $(38 a),(41 a) ;---$, Eqs. $(38 a, b),(39 a, b)$, using $\lambda_{0}=0.22$ from Run C1DV.

more complex in the presence of a mean distortion, which causes a variation in direction of the time-dependent wavevector; in the pressure-released case, the angular distribution of spectral energy is unchanged with respect to (isotropic) initial data, but the wavevector tends to be aligned with the symmetry (compression) direction (see (36)) so that $\cos ^{2} \alpha$ increases and tends to 1 . On the other hand, in the pure solenoidal limit, the relative concentration of spectral energy in the plane normal to the compression direction opposes the tendency induced by the wavevector motion so that a slower (as compared to the pressure-released case), but still positive, net increase of the anisotropy is obtained. Note that the solenoidal ratio of $D_{11}^{s} / q_{3}^{2}$ given by the DNS is found to be slightly lower than the RDT analytical prediction.

The cross-correlation $\left\langle u_{1}^{s} u_{1}^{d}\right\rangle /\left\langle u_{1}^{s} u_{1}^{s}\right\rangle$ is plotted in Figure 8 and compared to the RDT expression $C_{p}(J) / B_{s}(J)$ from (39a) and (40b). The results suggest that for modeling purposes it might be advantageous to use an effective "saturated" volumetric ratio $J^{+}$in place of $J$ and define $C_{p}^{+}$, an interpolating function for the cross-correlation, according to

$$
\frac{C_{p}^{+}(J)}{B_{s}^{+}(J)}=\frac{C_{p}\left(J^{+}\right)}{B_{s}\left(J^{+}\right)}
$$




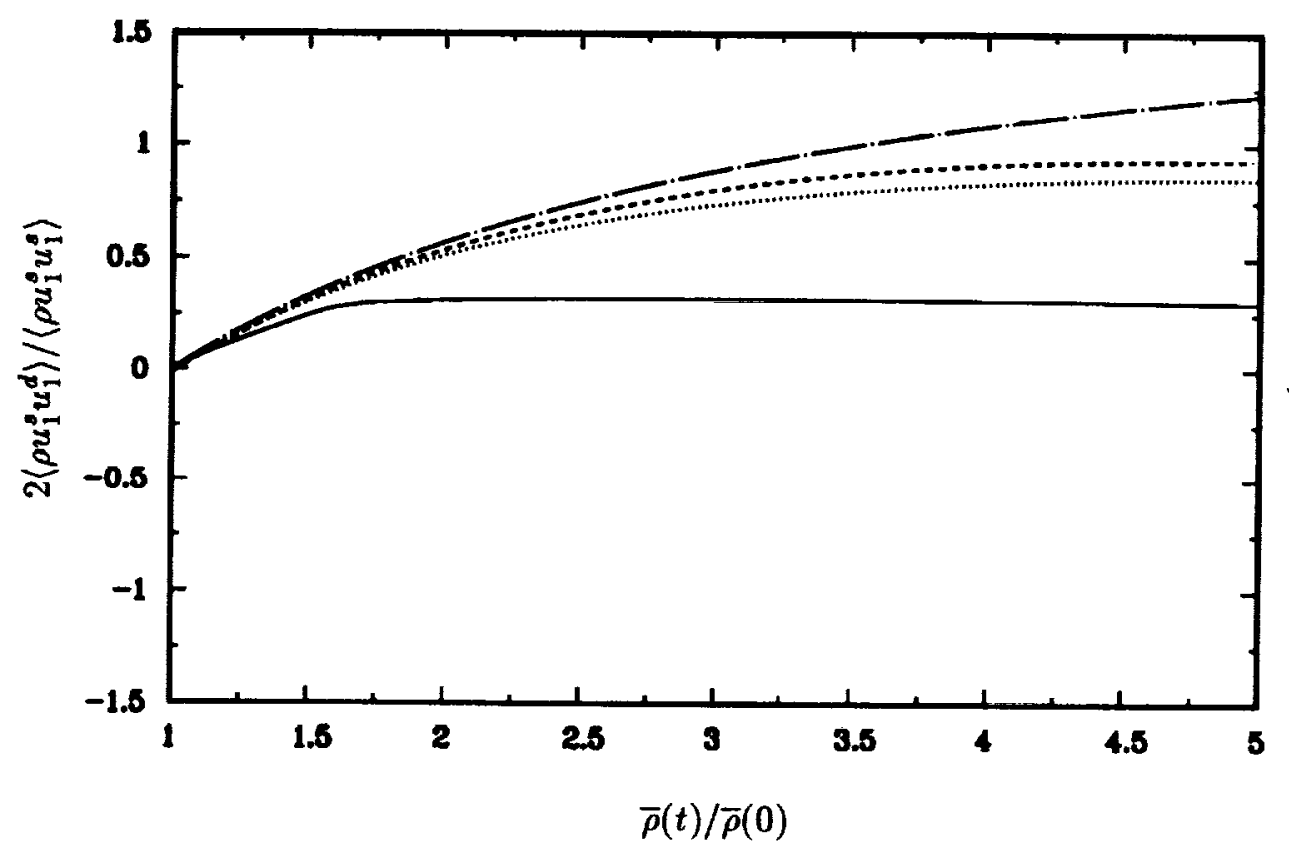

FiguRE 8. Histories of the compression-direction component of the dilatationalsolenoidal Reynolds stress correlation: - C1DJ $\left(M_{t}, \Delta m\right)_{t=0}=(0.03,0.3)$; --- , C1DV $(0.1,7) ; \cdots \cdots \cdot . \cdot$ C1DW $(0.3,1) ;-.-$, Eqs. $(39 a),(40 b)$.

and use the model

$$
\left\langle u_{1}^{s} u_{1}^{d}\right\rangle=C_{p}\left(J^{+}\right) q_{s}^{2}(0) .
$$

The parameter $J^{+}$would tend toward the actual $J$ in the pressure-released limit and approach unity in the solenoidal limit. The role of pressure will be discussed further in $\$ 4$; for now, we observe in Figures 9 and 10 the dramatic increase of both pressure variance and pressure-dilatation terms caused by the compression. The amplification increases with the initial turbulent Mach number, which at first seems to conflict with the idea of a pressure released limit. The paradox disappears, however, if the pressure-dilatation term is no longer nondimensionalised by initial values (as is done in Figures 9 and 10), but rather scaled by a term proportional to the kinetic energy production. DNS results for $\Pi / D q^{2}$ are presented on Fig. 11. The magnitude of this term is found to decrease with increasing $\Delta m$ for the three cases considered. This implies a non-monotonic variation with $\Delta m$ for this term (since it is identically zero in the solenoidal limit) with a maximum reached at low compressibility. It can be noticed that increasing values of $\Pi / D q^{2}$ are found at large $J^{-1}$ for the intermediate $\Delta m$ case (C1DW), which we expect cannot be explained by RDT. This illustrates that the requirements for a compression to be 


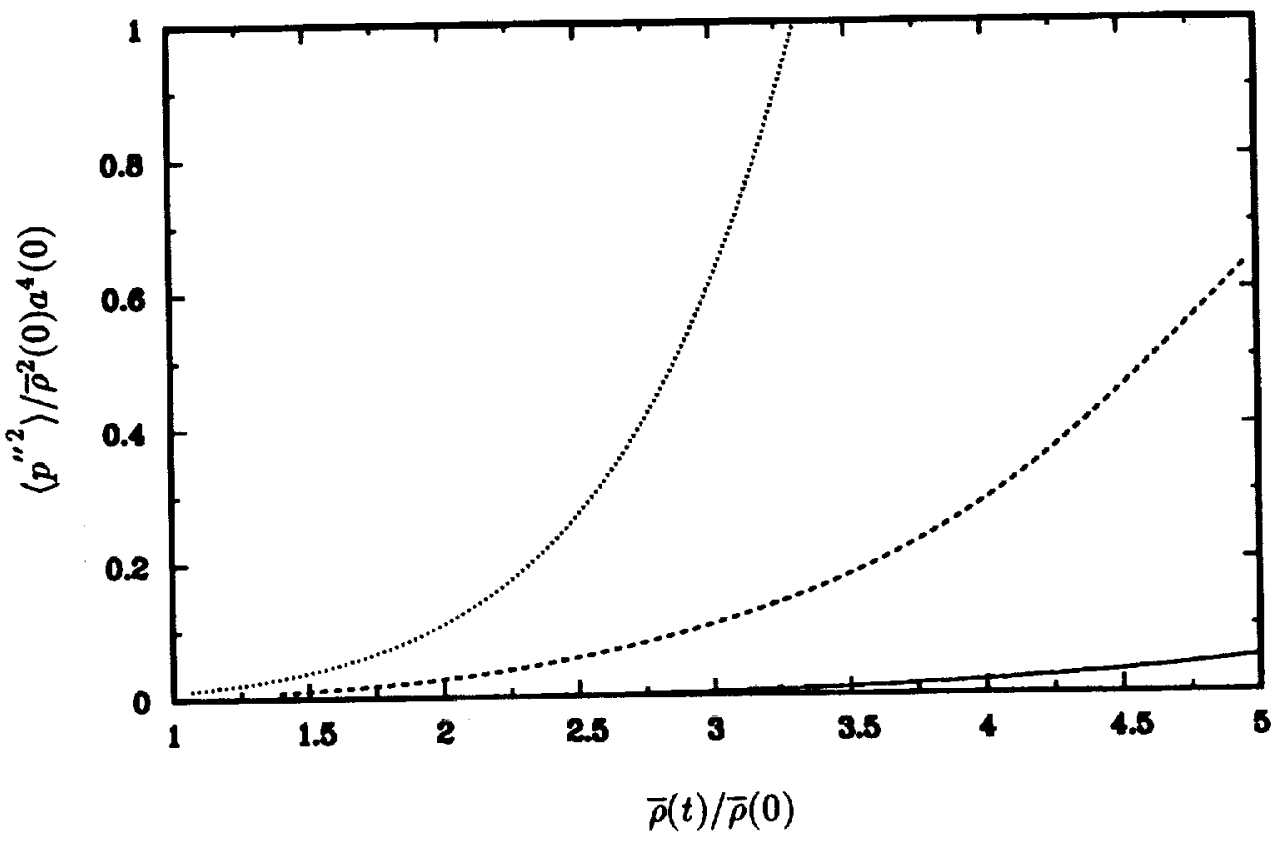

Figure 9. Pressure variance histories: - C1DJ $\left(M_{t}, \Delta m\right)_{t=0}=(0.03,0.3)$; $\ldots$, C1DV $(0.1,7) ; \cdots \cdots \cdots$, C1DW $(0.3,1)$.

rapid enough for RDT to be valid are more difficult to meet when the flow is intrinsically compressible, a fact also stressed by Zeman \& Coleman (1992). The term $\Pi_{11} / D q^{2}$ linked to the compression-direction component of the pressure-strain rate correlations is shown in Figure 12. The solenoidal RDT expression, $E_{s}(J) / A_{0}(J)$, from (38a) and (42a) is plotted and is found to give an upper limit to the DNS curves. These results suggest a monotonic decrease of $\Pi_{11} / D q^{2}$ with increasing $\Delta m$. Moreover, comparisons of the order of magnitude for both terms on Figures 11 and 12 (noting the different scales of the two plots) show that the compressiondirection component of the pressure-strain rate is dominant compared to its trace (pressure-dilatation term) in all cases. This confirms that the reduction of amplification of turbulent kinetic energy with respect to the pressure-released case (where only the "production" effects are present) is mainly due to $\Pi_{11}$, through reduction of anisotropy, as in the pure solenoidal case.

\section{Towards a pressure-strain rate model}

Equations for $\Pi_{11}$ and $\Pi$, valid for the rapid mean compression case, can be derived from eqs. (44) and (45), using eqs. (49) and (50) to model $q^{2}$ and $\left\langle u_{1} u_{1}\right\rangle$. 


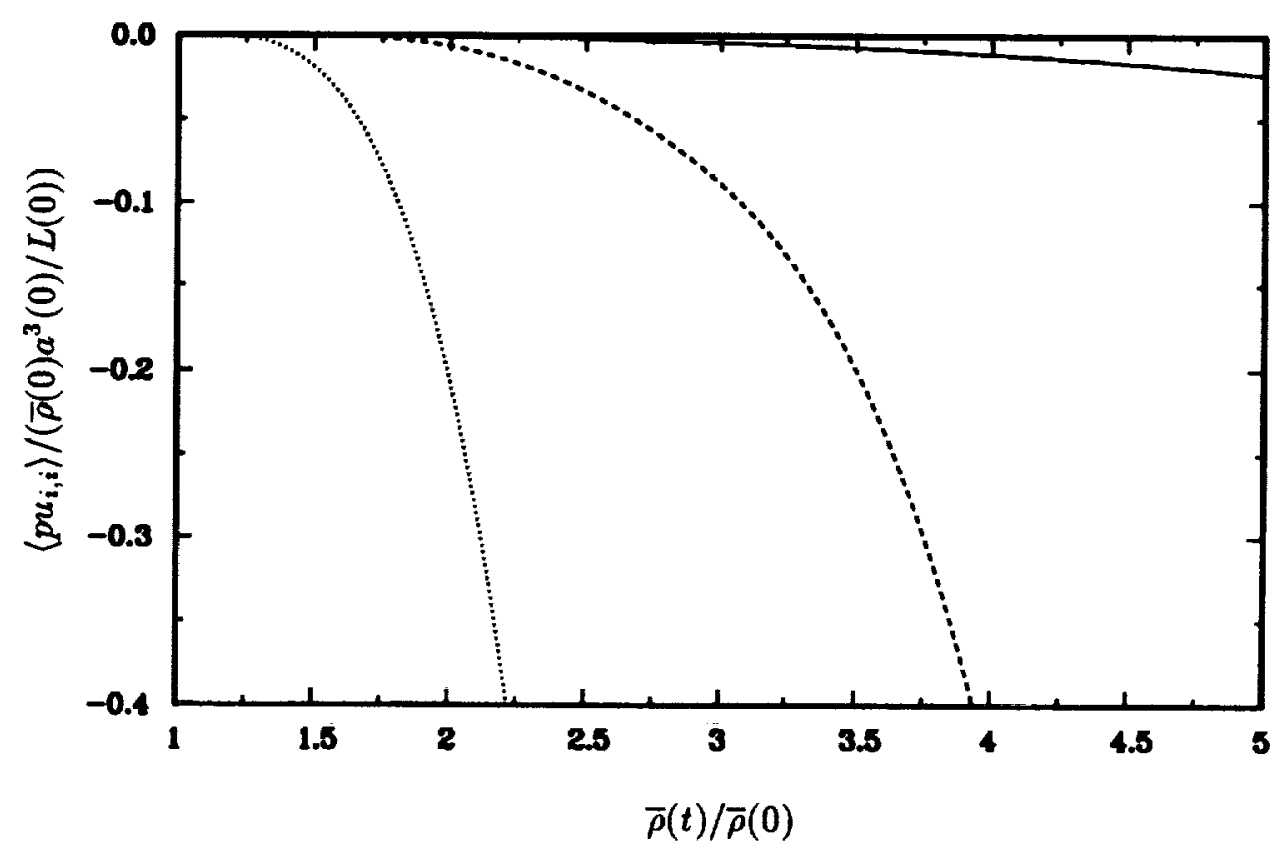

FIgURE 10. Pressure-dilatation correlation histories:,$- \operatorname{C1DJ}\left(M_{t}, \Delta m\right)_{t=0}=$ $(0.03,0.3) ;----$, C1DV $(0.1,7) ; \cdots \cdots \cdots$, C1DW $(0.3,1)$.

The result is

$$
\begin{gathered}
\Pi_{11}=J^{-2} \frac{d}{d t}\left(J^{2} R_{11}\right) \\
=J^{-2} \frac{d}{d t}\left(J^{2}\left(B_{s}+B_{p}^{+}-B_{s}^{+}\right) q_{s}^{2}(0)+J^{2} B_{p} q_{d}^{2}(0)\right) \\
=D E_{s} q_{s}^{2}(0)+J^{-2} \frac{d}{d t}\left(J^{2}\left(B_{p}^{+}-B_{s}^{+}\right)\right) q_{s}^{2}(0)
\end{gathered}
$$

and

$$
\begin{gathered}
\Pi=\left(\frac{1}{2}\left(\dot{A}_{s}+\dot{A}_{p}^{+}-\dot{A}_{a}^{+}\right)+D\left(B_{s}+B_{p}^{+}-B_{s}^{+}\right)\right) q_{s}^{2}(0) \\
=\left(\frac{1}{2}\left(\dot{A}_{p}^{+}-\dot{A}_{s}^{+}\right)+D\left(B_{p}^{+}-B_{s}^{+}\right)\right) q_{s}^{2}(0) .
\end{gathered}
$$

To obtain the above, the relations

$$
J^{-2} \frac{d}{d t}\left(J^{2} B_{s}\right)=E_{s} ; \frac{d}{d t}\left(J^{2} B_{p}\right)=0 ; \frac{1}{2} \dot{A}_{s}+D B_{s}=0 ; \frac{1}{2} \dot{A}_{p}+D B_{p}=0
$$

have also been used. 


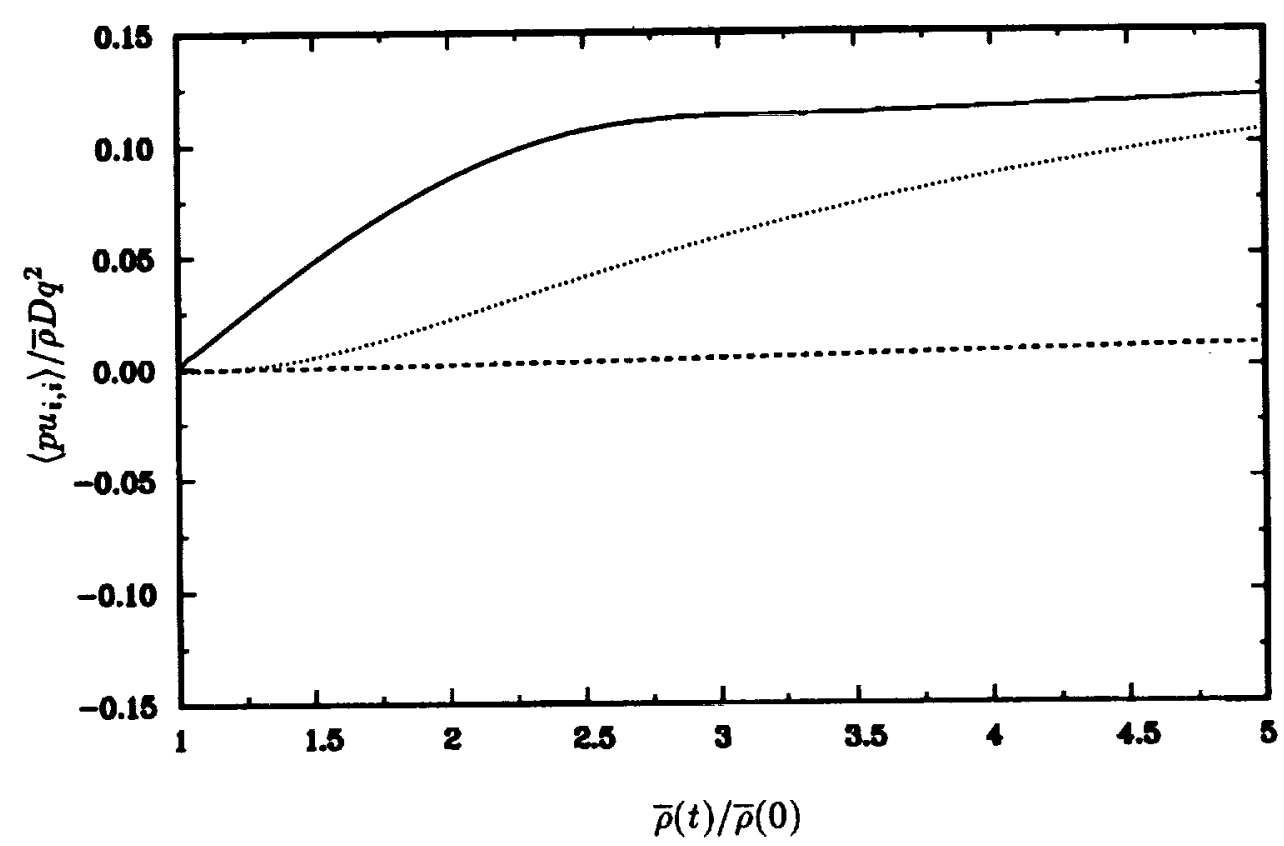

FIGURE 11. Rescaled pressure-dilatation correlation histories: - C1DJ $\left(M_{t}, \Delta m\right)_{t=0}=(0.03,0.3) ;-\cdots, \operatorname{C1DV}(0.1,7) ; \cdots \cdots \cdots, \operatorname{C1DW}(0.3,1)$.

\subsection{Proposals for Second-Order Modeling}

Two simple ideas for constructing the Eq. (52) and (53) "interpolation" functions (denoted by a superscript "+") are proposed:

1. Using two functions of $\Delta m$, passing monotonically from from 0 to 1 so that $A_{p}^{+}-A_{s}^{+}=f_{1}\left(A_{p}-A_{s}\right)$ and $B_{p}^{+}-B_{s}^{+}=f_{2}\left(B_{p}-B_{s}\right)$; if the time-variation of the interpolation functions is neglected, this leads to the model

$$
\begin{gathered}
\Pi_{11}=\Pi_{11}^{s}\left(1-f_{2}\right) \\
\Pi=\left(f_{2}-f_{1}\right) D\left(B_{p}-B_{s}\right) q_{s}^{2}(0) .
\end{gathered}
$$

Note that $f_{2}>f_{1}$ is consistent with the sign of $\Pi$ found in the DNS results and with the interpretation of dilatational energy histories in Figure 5.

2. Using a "saturated" volumetric ratio $J^{+}$instead of the actual $J$ in the evaluation of the interpolation functions with + superscripts, so that $A^{+}(J)=A\left(J^{+}\right)$. The equation for $J^{+}$would be

$$
j^{+}=U_{i, i} J^{+}-C_{J+} \frac{a}{L}\left(J^{+}-1\right)
$$




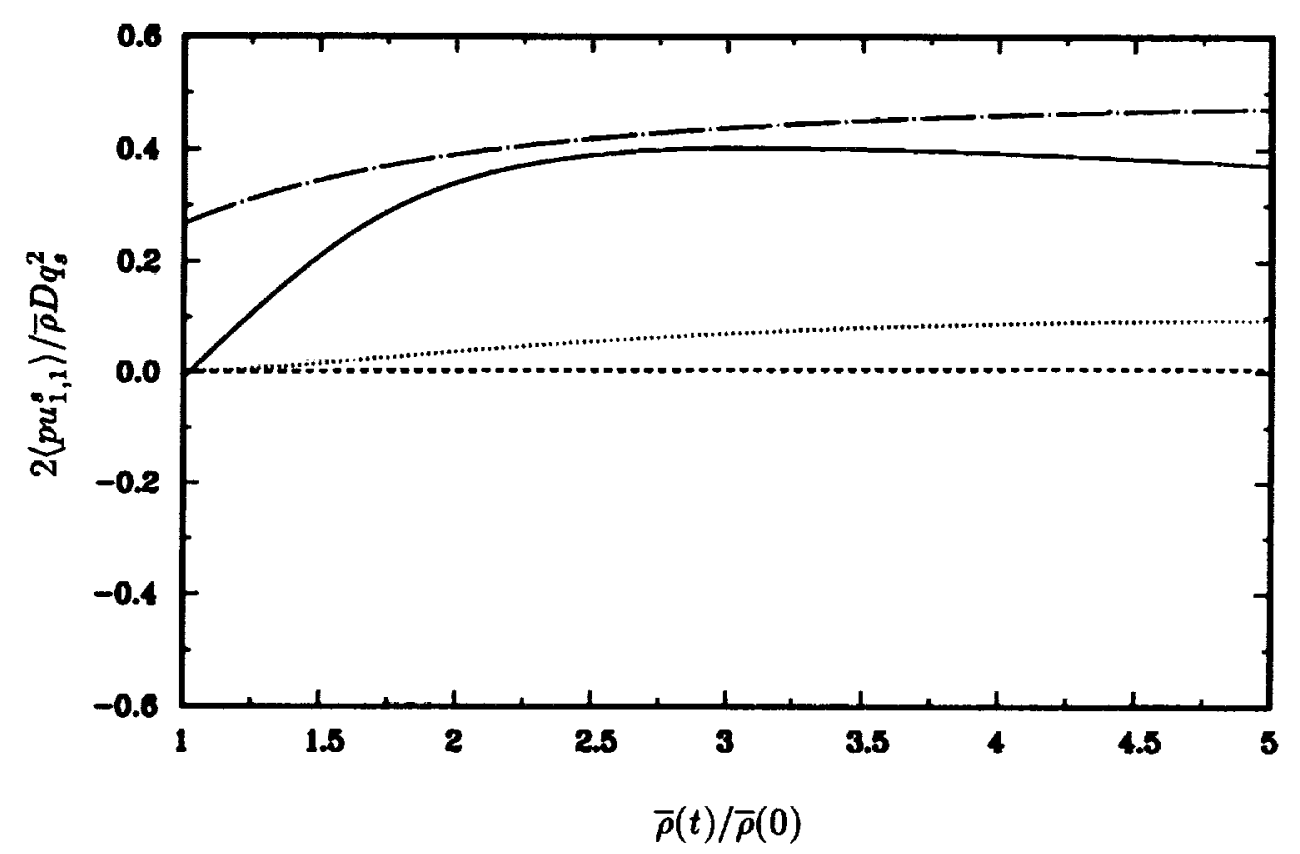

FIGURE 12. Histories of correlation of pressure and compression-direction velocity gradient: $\longrightarrow, \operatorname{C1DJ}\left(M_{t}, \Delta m\right)_{t=0}=(0.03,0.3) ; \cdots$, C1DV $(0.1,7) ; \cdots \cdots \cdots$, C1DW $(0.3,1) ;--$, Eqs. $(38 a)$ and $(42 a)$.

where $C_{J+}$ is a modeling constant. The sonic timescale-damping term would allow $J^{+}$to saturate close to unity as the regime of the flow approaches the solenoidal limit.

\subsection{Testing a Second-Order Model}

From our analysis of the three DNS cases, we find that they are in the regime where the production and the rapid redistribution terms are dominant. The contribution of the pressure-dilatation is about $10 \%$ of the production in the worst case. This leads us in our attempt to model the DNS results to adopt the first proposal of the previous subsection, and consider a linear (in $b_{i j}$ ) model for the solenoidal rapid part (see Shih et al. , 1990) of the redistribution term, taking $1-f_{2}$ (see Eq. $(55 a)$ ) to be an exponential function of $\Delta m$. The mean and Reynolds-stress equations reduce to:

$$
\begin{gathered}
\bar{\rho}_{, \ell}=-U_{i, i} \bar{\rho}, \quad U_{i, j}=\frac{D_{0}}{1+D_{0} t} \delta_{i 1} \delta_{j 1}, \quad T_{, t}=-(\gamma-1) T U_{i, i} \\
R_{i j, t}=-R_{i k} U_{j, k}-R_{j k} U_{i, k}+\Phi_{i j} \exp \left(-\Delta m / C_{\Delta m}\right)
\end{gathered}
$$




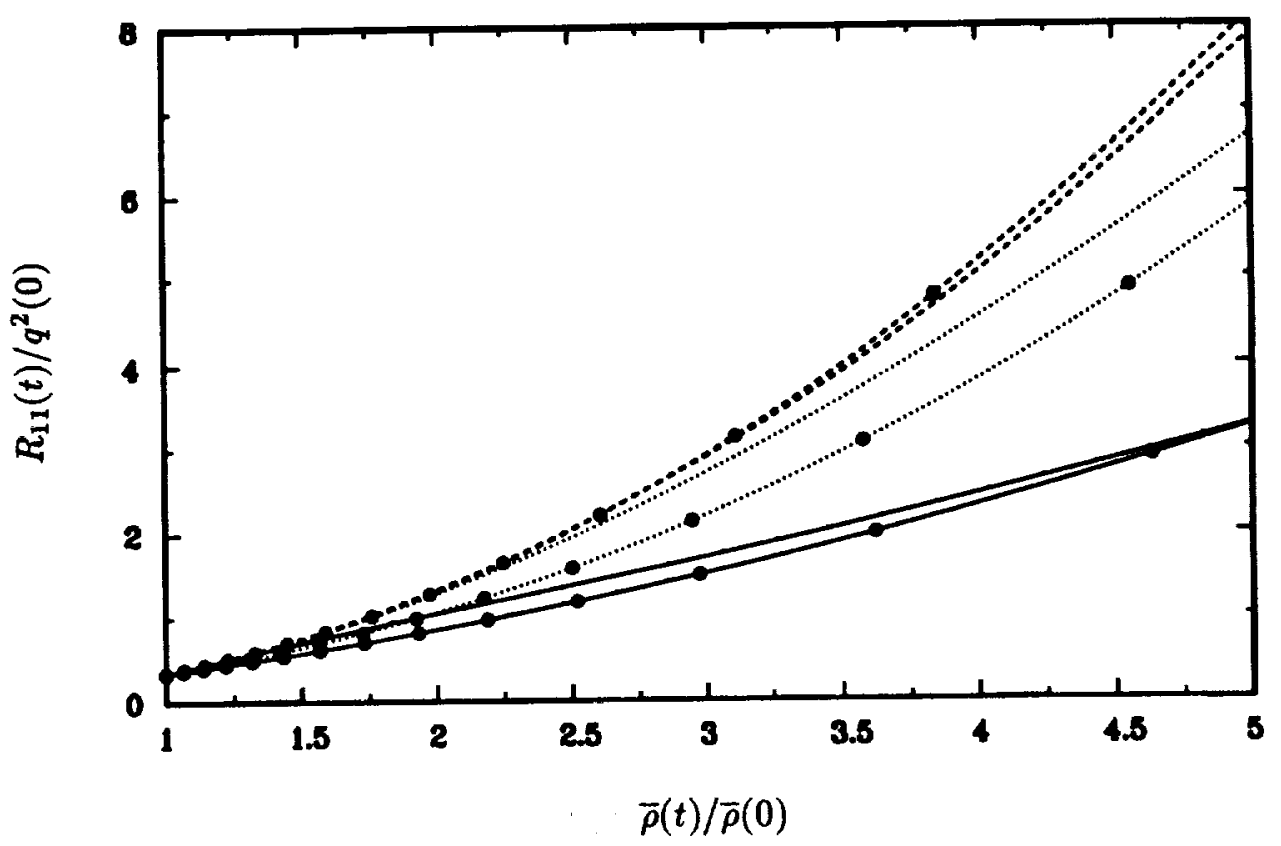

FIGURE 13. Reynolds stress history, compression-direction component: - , C1DJ $\left(M_{t}, \Delta m\right)_{t=0}=(0.03,0.3) ;-\cdot--$, C1DV $(0.1,7) ; \cdots \cdots$, C1DW $(0.3,1) ; \bullet$, Eq. (55d); no symbols, DNS.

with

$$
\begin{aligned}
\frac{\Phi_{i j}}{\frac{1}{2} q^{2}}=\frac{4}{5} S_{i j}^{*}+ & \frac{48 \alpha-60}{35}\left(S_{i k}^{*} b_{k j}+S_{j k}^{*} b_{k i}-\frac{2}{3} S_{m n}^{*} b_{m n} \delta_{i j}\right) \\
& +\frac{60-16 \alpha}{15}\left(\Omega_{k i} b_{k j}+\Omega_{k j} b_{k i}\right),
\end{aligned}
$$

where $\Delta m=\sqrt{S_{i j} S_{j i}} M_{t} q^{2} / \epsilon$ and we have set $\alpha=2.523$ (to be consistent with the model of Launder et al. (1975)), and $C_{\Delta m}=40$. The quantity $\Omega_{i j}=\left(U_{i, j}-U_{j, i}\right) / 2$ is the mean rotation tensor.

The development of the axial component of the Reynolds stress, $R_{11}$, as predicted by the above model for the three cases considered is shown in figure 13 . We find that this simple model, where the effects of the redistributive term diminish when $M_{t}$ increases, compares well with the DNS data. The development of the turbulent kinetic energy (see Fig. 14) is also well reproduced, indicating that the effects of the pressure-dilatation are, in fact, weak compared to the production term. No attempt was made to optimize the constant $C_{\Delta m}$ since the pressure-dilatation term was neglected. This term does play a role in the development of the flow, and $C_{\Delta m}$ should be optimized in conjunction with a model for the pressure dilatation term. 


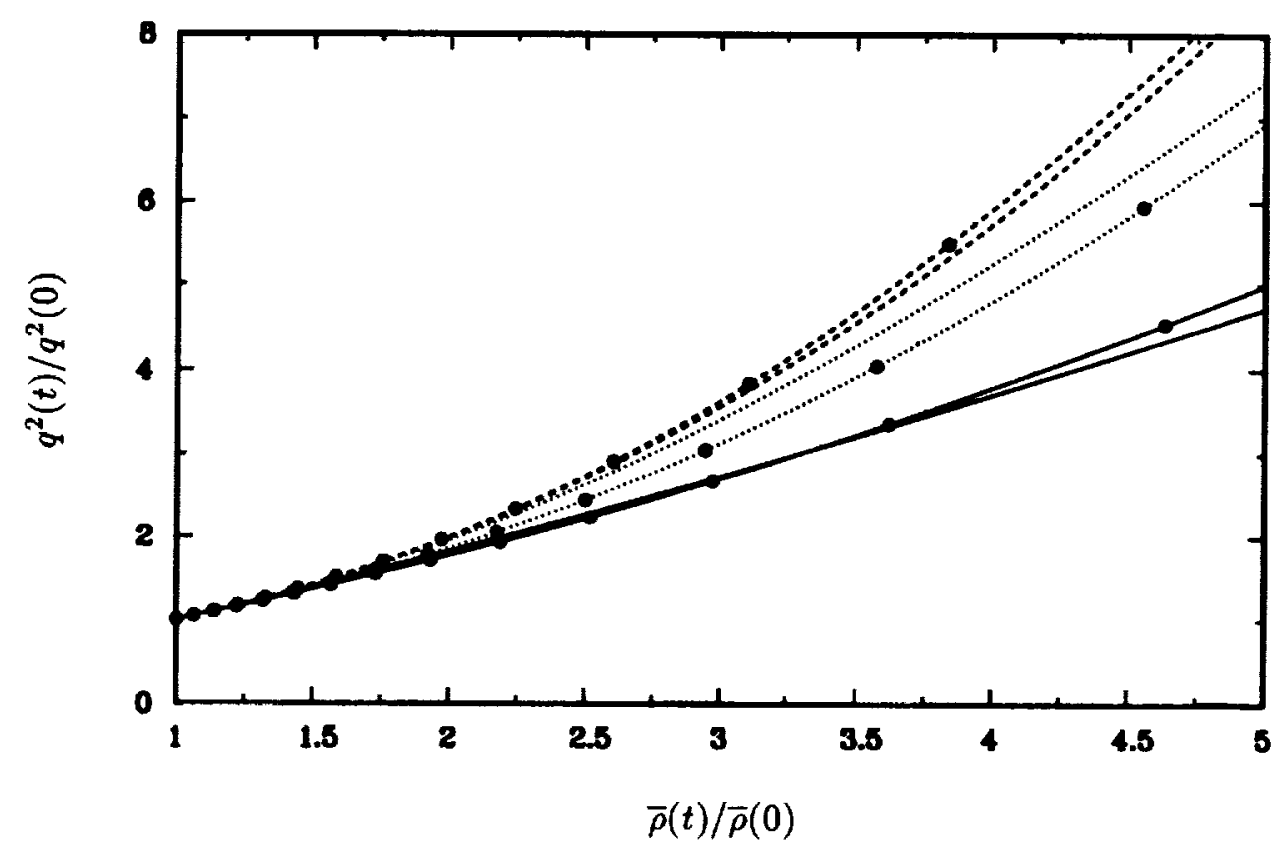

FIGURE 14. Turbulent kinetic energy history:,$- \operatorname{C1DJ}\left(M_{t}, \Delta m\right)_{t=0}=(0.03$, $0.3) ;-\cdots$, C1DV $(0.1,7) ; \cdots \cdots \cdots$, C1DW $(0.3,1)$; •, Eq. $(55 d)$; no symbols, DNS.

\section{Spherical compression and pure shear revisited}

\subsection{Isotropic spherical compression}

In the presence of a mean spherical compression, with

$$
U_{i, j}=D \delta_{i j}, \quad D=\frac{D_{0}}{1+D_{0} t}=D_{0} J^{-1 / 3} F_{i j}=J^{1 / 3} \delta_{i j} \quad \text { and } \quad k_{i}=K_{i} J^{-1 / 3} \text {, }
$$

the coupling term $m_{3 \alpha}$ in (27) and (28) has zero value. The evolution of the solenoidal kinetic energy is then easily found to be given by the amplification coefficient $J^{-2 / 3}$. For the dilatational field, eqs. (33) and (34) remain of interest now with their right-hand sides equal to zero (since $\widehat{p} \sim m_{3 \alpha}$ ). Even in the absence of the right-hand sides, a WKB analysis of the equations would not in general be appropriate because the timescale variation of $a^{2}$ and $k^{2}$ is not necessarily small with respect to the expected frequency $a k$ of the oscillating system (depending on the value of $\Delta m$ ). Blaisdell (1992, private communication) has recently found a solution free of WKB assumptions; its validity is restricted to values of $\gamma$ close to $5 / 3$, but a general analytical solution is possible (work in progress). If $\gamma=5 / 3$, $k^{2}$ and $a^{2}$ have the same $J^{-2 / 3}$ time dependence, so simple solutions in terms of $\exp ( \pm \mathrm{i} a(0) k(t) t)$, where $k(t)$ varies as in (56), can be obtained for $y$ and $z$. The 
history of $q_{d}^{2}$ can then be derived from the initial (uncompressed) dilatational field. Assuming that acoustic equilibrium holds for the initial conditions, one can write

$$
q_{d}^{2}(t)=J^{-2 / 3} q_{d}^{2}(0)
$$

which is the same variation found for the solenoidal energy. The acoustic equilibrium assumption is realistic but perhaps not necessary; the initial balance between kinetic dilatational energy and potential (pressure) energy allows oscillating terms to be dropped, but the same final result could also be reached after a certain elapsed time because of the damping behavior of integrals such as

$$
\int F(K) \exp \left(2 \mathrm{i} a(0) K J^{-1 / 3} t\right) d K,
$$

where $F$ is defined by the initial energy spectra and is nonzero only for flows out of acoustic equilibrium. (This behavior is similar to that found for the case of rapid rotation.)

The above considerations show that an oscillating regime, more general than the pure acoustic one, is not inconsistent with the pressure-released limit and that the latter can be used to derive the same relationship (57) found via the acoustic equilibrium assumption. We thus find that the spherically compressed flow lends support to the general approach advocated in this paper.

\subsection{Pure plane shear}

The case of shear flow is particularly interesting because all the coupling terms, most notably $m_{\alpha 3}$ and $m_{3 \alpha}$, are present. The crucial parameter in the absence of compression $(J=1)$ is the shear $S=d U_{1} / d x_{2}$. Under this deformation, eqs. (27), (33) and (34) become

$$
\begin{gathered}
\dot{\hat{\varphi}}^{(1)}+S \frac{k_{3}}{k} \widehat{\varphi}^{(2)}=S\left(\frac{k_{3} k_{2}}{\left(k_{1}^{2}+k_{3}^{2}\right)^{1 / 2}}\right) y \\
\frac{\mathcal{D}}{\mathcal{D} t}\left(k \hat{\varphi}^{(2)}\right)=-S\left(\frac{k_{1}}{\left(k_{1}^{2}+k_{3}^{2}\right)^{1 / 2}}\right) k^{2} y \\
\ddot{y}+a_{0}^{2} k^{2} y+S^{2} \frac{k_{1}^{2}}{k^{2}} y=S^{2} \frac{\mathcal{D}}{\mathcal{D}(S t)}\left(\frac{k_{1}\left(k_{1}^{2}+k_{3}^{2}\right)^{1 / 2}}{k^{3}}\right) k \hat{\varphi}^{(2)}
\end{gathered}
$$

with

$$
U_{i, j}=S \delta_{i 1} \delta_{j 2}, k_{1}=K_{1}, k_{2}=K_{2}-K_{1} S t \text { and } k_{3}=K_{3} .
$$

Here the polar axis is chosen to be in the gradient direction $\left(n_{i}=\delta_{i 2}\right)$. The two solenoidal $\hat{\varphi}^{(1)}$ and $\hat{\varphi}^{(2)}$ components are very close to the set $\left(\omega_{2}, \nabla^{2} u_{2}\right)$ used in linear stability analyses for decoupling, for example, the Orr-Sommerfeld equations for parallel flows (cf. Waleffe 1990). Even in the pure solenoidal case (where $y=$ $\left.\hat{\varphi}^{(3)} / k=0\right)$, the present approach appears to be more tractable than are classic RDT approaches (Townsend 1976). Unlike for a purely irrotational mean deformation, 
the presence of the new coupling terms (mediated by $m_{13}=-S k_{2} k_{3} / k k_{13}, m_{23}=$ $S k_{3} / k_{13}$ in the above equations) makes the solenoidal field no longer independent of the dilatational component. In addition, this coupling introduces the new term $S^{2}\left(k_{1}^{2} / k^{2}\right) y$ in Eq. (59). The pressure-released approximation amounts to neglecting $a_{0}^{2} k^{2} y$ compared both to this new term and to the solenoidal right-hand side in (59). The $\Delta m \gg 1$ regime then implies that (in physical space),

$$
u_{1}=u_{1}(0)-S t u_{2}(0), u_{2}=u_{2}(0) \text { and } u_{3}=u_{3}(0) \text {, }
$$

and leads to quadratic amplification, with respect to $S t$, of the kinetic energy (which is more rapid than the nearly linear amplification obtained by numerically integrating the solenoidal RDT solution for $\frac{1}{2}\left\langle\widehat{\varphi}^{(1) \star} \widehat{\varphi}^{(1)}+\widehat{\varphi}^{(2) \star} \widehat{\varphi}^{(2)}\right\rangle$ over k-space). Note that the inviscid solenoidal RDT solution for the vertical velocity component is given by $\mathcal{D}\left(\nabla^{2} u_{2}\right) / \mathcal{D} t=0$ in physical space (corresponding to Eq. (58) with $y=0$ ) so that a rapid decay of $u_{2}$ is found. On the other hand, $u_{2}$ is conserved in the pressure-released inviscid RDT limit.

\section{Recap and conclusions}

The objective of this analysis has been to develop a rapid distortion theory for homogeneous compressible turbulence at finite Mach number and then use that theory to explore some issues related to one-point compressible turbulence models. We have applied the analysis to the case of axial compression and found that DNS results confirm the RDT prediction of two distinct flow regimes, one for vanishingly small turbulent Mach number and the other for flows with negligible sonic and turbulent timescale variations compared to the mean distortion. The latter is referred to as the pressure-released regime (since the fluctuating pressure field can be neglected in the RDT for this limit) and is defined by large values of the product of $M_{t}$ and the ratio of the turbulent to mean deformation timescales. For large values of this parameter, we find that the intrinsic compressibility of the turbulence is responsible for an increase in the growth rate of kinetic energy with increasing $M_{t}$, an effect exactly opposite to that usually attributed to the compressibility. It would seem that the reduction in kinetic energy growth rate due to compressibility observed in previous compressible homogeneous DNS studies can be attributed to "slow" terms with nonlinear and dissipative origin, such as the "extra" dilatational dissipation associated by Zeman (1990) with eddy "shocklets." In the future, we plan to perform systematic comparisons between compressible RDT (from numerical solutions obtained by the method presented in $\S \S 2.4$ ) and existing DNS to allow an accurate differentiation between the "rapid" and "slow" terms, which are found to have opposite trends with respect to the effect of compressibility on the kinetic energy growth rate.

For the axial compression, analytic expressions for the correlations associated with one-point closures for both the solenoidal and pressure-released limits have been given. These expressions have been used to propose methods of interpolating between the two limiting RDT cases in models for the pressure-strain rate correlation, $\Pi_{i j}$ and thus account for finite Mach number effects. 
All the DNS results were obtained using the facilities of the Numerical Aerodynamic Simulation program. This work was partially supported by the Laboratoire de Mécanique des Fluides et d'Acoustique, Ecole Centrale de Lyon.

\section{REFERENCES}

Bataille, F., Bertoglio J. P. \& MARION, J. D. 1992 Spectral study of weakly compressible isotropic turbulence. To appear in $C$. R. Académie des Sciences, Paris.

Batchelor, G. K. \& Proudman, I. 1954 The effect of rapid distortion on a fluid in turbulent motion. Q. J. Mech. Appl. Math. 7, 83.

Blaisdell, G. A., Mansour N. N. \& Reynolds, W. C. 1991 Numerical simulation of compressible homogeneous turbulence. Department of Mechanical Engineering, Stanford University, Thermosciences Division Report TF-50.

CAMBON, C. 1982 Etude spectrale d'un champ turbulent incompressible soumis à des effets couplés de déformation et de rotation imposés extérieurement. thèse d'Etat. Université Claude-Bernard-Lyon I.

CAMBon, C 1990 Single and double point modeling of homogeneous turbulence. CTR Annual Research Briefs. Stanford University/NASA Ames.

Cambon, C., Teissèdre, C. \& Jeandel, D. 1985 Etude d'effets couplés de déformation et de rotation sur une turbulence homogène. J. Méc. Théor. Appl. 4,629 .

CAMBON, C. \& JACQUIN, L. 1989 Spectral approach to non-isotropic turbulence subjected to rotation. J. Fluid Mech. 202, 295.

Cambon, C., Jacquin, L. \& Lubrano, J. L. 1992 Toward a new Reynolds stress model for rotating turbulent flows. Phys. Fluids A. 4(4), 812.

Coleman, G. N. \& Mansour, N. N. 1991 Modeling the rapid spherical compression of isotropic turbulence. Phys. Fluids A. 3(9), 2255.

CraYA, A. 1958 Contribution à l'analyse de la turbulence associée à des vitesses moyennes P.S.T. No. 345.

Debiève, J. F., Gouin H. \& Gaviglio J. 1982 Evolution of the Reynolds stress tensor in a shock wave turbulence interaction. Indian J. Tech. 20, 90.

Durbin, P. A. \& ZEMAN, O. 1992 Rapid distortion theory for homogeneous compressed turbulence with application to modeling. To appear in J. Fluid Mech.

Eringen, A. C. 1967 Mechanics of Continua, Wiley.

GoldSTEIN, M. E. 1978 Unsteady vortical and entropic distortions of potential flows round arbitrary obstacles. J. Fluid Mech. 89, 433.

Greenspan, H. P. 1968 The Theory of Rotating Fluids, Cambridge University Press. 
Herring, J. R. 1974 Approach of axisymmetric turbulence to isotropy. Phys. Fluids. 17, 859.

JACQUIN, L. \& CAMBON, C. 1992 Turbulence amplification by a shock wave and rapid distortion theory. submitted to Phys. Fluids $A$.

Launder, B. E., Reece, G. J. \& Rodi, W. 1975 Progress in the development of a Reynolds-stress turbulence closure. J. Fluid Mech.. 68, 537.

LEE, S., LELE, S. K. \& MoIN, P 1992 Interaction of isotropic turbulence with a shock wave. Department of Mechanical Engineering, Stanford University, Thermosciences Division Report TF-52.

Mansour N. N., Shin T-H. \& Reynolds, W. C. 1991 The effects of rotation on initially anisotropic homogeneous flows. Phys. Fluids A. 10, 2421.

REYNolds, W. C. 1990 Towards a structure-based turbulence model. Special workshop for turbulence modelers, CTR summer program.

REYNOLDS, W. C. 1992 Towards a structure-based turbulence modeling. Studies in Turbulence. Editors Gatski et al. (Springer-Verlag, New York) 76.

RiBNER, H. S. 1953 Convection of a pattern of vorticity through a shock wave. NACA Report 1164.

Rogallo, R. S. 1981 Numerical experiments in homogeneous turbulence. NASA TM 81315.

SABEL'NIKOV, V. A. 1975 Pressure fluctuations generated by uniform distortion of homogeneous turbulence. Fluid Mech. Soviet Res. 4, 46.

Sarkar, S., Erlebacher, G., Hussaini, Y. M. \& Kreiss, H. O. 1989 The analysis and modeling of dilatational terms in compressible turbulence. ICASE Report 89-79.

Townsend, A. A. 1976 The Structure of Homogeneous Turbulence, Cambridge University Press.

WALEFFE, F. 1990 On the origin of the streak spacing in turbulent shear flows. CTR Annual Research Briefs. Stanford, Univ./NASA Ames, 159.

WALEFFE, F. 1992 Inertial transfers in the helical decomposition. Submitted to Phys. Fluids A.

ZEMAN, O. 1990 Dilatational dissipation: the concept and application in modeling compressible mixing layers. Phys. Fluids A. 2, 178.

Zeman, O. \& Coleman, G. N. 1992 Compressible turbulence subjected to shear and rapid compression. Eight Symposium on Turbulent Shear Flows, Munich. Springer-Verlag. 